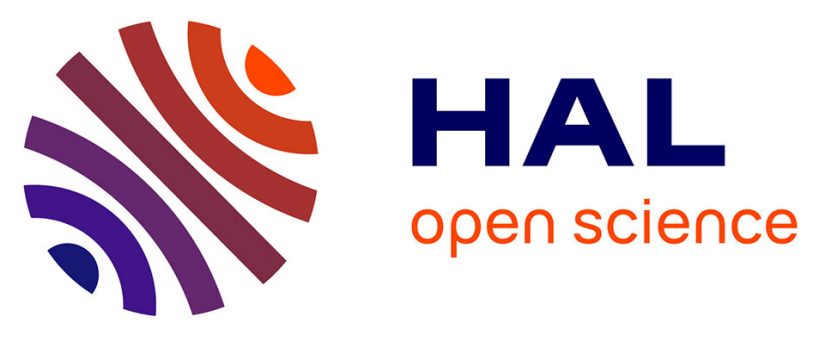

\title{
Setting Carriers Free: Healing Faulty Interfaces Promotes Delocalization and Transport in Nanocrystal Solids
}

\author{
Willem Walravens, Eduardo Solano, Filip Geenen, Jolien Dendooven, Oleg \\ Gorobtsov, Athmane Tadjine, Nayyera Mahmoud, Patrick Peiwen Ding, Jacob \\ Ruff, Andrej Singer, et al.
}

\section{To cite this version:}

Willem Walravens, Eduardo Solano, Filip Geenen, Jolien Dendooven, Oleg Gorobtsov, et al.. Setting Carriers Free: Healing Faulty Interfaces Promotes Delocalization and Transport in Nanocrystal Solids. ACS Nano, 2019, 13 (11), pp.12774-12786. 10.1021/acsnano.9b04757 . hal-02517025

\section{HAL Id: hal-02517025 \\ https://hal.science/hal-02517025}

Submitted on 12 Dec 2020

HAL is a multi-disciplinary open access archive for the deposit and dissemination of scientific research documents, whether they are published or not. The documents may come from teaching and research institutions in France or abroad, or from public or private research centers.
L'archive ouverte pluridisciplinaire HAL, est destinée au dépôt et à la diffusion de documents scientifiques de niveau recherche, publiés ou non, émanant des établissements d'enseignement et de recherche français ou étrangers, des laboratoires publics ou privés. 


\title{
Setting Carriers Free: Healing Faulty Interfaces Promotes Delocalization and Transport in Nanocrystal Solids
}

Willem Walravens, Eduardo Solano, Filip Geenen, Jolien Dendooven, Oleg Gorobtsov, Athmane Tadjine, Nayyera Mahmoud, Patrick Peiwen Ding, Jacob P. C. Ruff, Andrej Singer, Gunther Roelkens, Christophe Delerue, Christophe Detavernier, and Zeger Hens

Physics and Chemistry of Nanostructures (PCN), Ghent University, Gent, Belgium

Center for Nano and Biophotonics, Ghent University, Gent, Belgium

NCD-SWEET beamline, ALBA Synchrotron Light Source, Carrer de la Llum 2-26, 08290 Cerdanyola del

Vall es, Spain

Conformal Coating of Nanomaterials (CoCooN), Ghent University, Gent, Belgium

Department of Materials Science and Engineering, Cornell University, Ithaca, NY, 14850, USA

Université de Lille, CNRS, Centrale Lille, Yncrea-ISEN, UPHF, UMR 8520-IEMN, 59000 Lille, France

Photonics Research Group, Ghent University, Gent, Belgium

CHESS, Cornell University, Ithaca, NY, 14850, USA

E-mail: zeger.hens@ugent.be

Cite this: ACS Nano 2019, 13, 11, 12774-12786

https://doi.org/10.1021/acsnano.9b04757

\begin{abstract}
Superlattices of epitaxially connected nanocrystals (NCs) are model systems to study electronic and optical properties of NC arrays. Using elemental analysis and structural analysis by in situ X-ray fluorescence and grazing-incidence small-angle scattering, respectively, we show that epitaxial superlattices of PbSe NCs keep their structural integrity up to temperatures of $300^{\circ} \mathrm{C}$; an ideal starting point to assess the effect of gentle thermal annealing on the superlattice properties. We find that annealing such superlattices between 75 and $150{ }^{\circ} \mathrm{C}$ induces a marked red shift of the NC bandedge transition. In fact, the post-annealing band-edge reflects theoretical predictions on the impact of charge carrier delocalization in these epitaxial superlattices. In addition, we observe a pronounced enhancement of the charge carrier mobility and a reduction of the hopping activation energy after mild annealing. While the superstructure remains intact at these temperatures, structural defect studies through $\mathrm{X}$-ray diffraction indicate that annealing markedly decreases the density of point defects and edge dislocations. This indicates that the connections between NCs in as-synthesized superlattices still form a major source of grain boundaries and defects, which prevent carrier delocalization over multiple NCs and hamper NC-to-NC transport. Overcoming the limitations imposed by interfacial defects is therefore an essential next step in the development of high-quality optoelectronic devices based on NC solids.
\end{abstract}

KEYWORDS: nanomaterials self-assembly nanocrystal solids charge transport grain boundary nanocrystal-nanocrystal interface defects

Artificial solids made of ordered assemblies of nanocrystals (NCs) have sparked interest in various research fields, as such materials may exhibit properties-by-design through the choice of the NC building blocks.(1) Examples range from data storage in arrays of magnetic nanoparticles $(2,3)$ over detection of DNA and peptides(4) to the fabrication of thermometers and $\mathrm{pH}$ meters. (5) A case in 
point are superlattices of semiconductor NCs, which offer the appealing combination of a tunable band gap, high absorption coefficients, and a suitability for solution-based processing and find applications in, for example, photodetectors,(6-9) solar cells,(10-12) and field-effect transistors.(13-15) Such NC devices typically rely on NC films produced through, for example, spincoating, dropcasting, or spraycoating. This results in disordered NC stacks, in which excessive surface defects or restricted dot-to-dot hopping can compromise electronic transport. Strategies to overcome such limitations focused on exchanging the native ligands with shorter organic or inorganic moieties to decrease the interdot distance and passivate dangling bonds or trap states. $(9,14,16-18)$ Not only have these approaches steadily improved device performance, they also led researchers to discover methods for creating NC superlattices in which individual dots are epitaxially connected through covalent bonds.(19-25) Theoretical work predicts that such epitaxial superlattices exhibit an intricate electronic band structure that can be tuned by the choice of building block and the supercrystal symmetry.(26-29)

The elimination of any tunnelling barrier between adjacent NCs in an epitaxial superlattice is expected to yield high mobility, band-like charge transport. Nevertheless, while high carrier mobilities have been measured in such films, Whitham and co-workers showed that carrier transport in epitaxial superlattices of PbSe NCs still involved hopping of localized charge carriers.(24) This persistent localization of charge carriers was assigned to disorder, where especially coupling disorder, that is, variation in the interdot contact area, was put forward as a major factor preventing delocalization. Given the fact that superlattices are typically formed at room temperature or close to room temperature, however, one could also approach the interface connecting neighboring NCs as a grain boundary, rather than an epitaxial connection. In bulk metals, it is known that defects, vacancies or dislocations at grain boundaries can serve as trap or scattering sites that reduce the mobility of charge carriers. So far, the crystalline quality of the interfaces created in NC solids has received little attention, even if recent literature studies highlight the relation between nanocrystalline grain boundaries and material properties. Sanchez et al., for example, showed that line defects are stable in GaAsP nanowires and demonstrated through cathodoluminescence on individual nanowires that defective regions are responsible for the quenching of optical emission.(30) Ondry et al. used in situ high-resolution transmission electron microscopy (HRTEM) to detail the trajectory through which dislocations between misaligned $\mathrm{PbTe} N \mathrm{NS}$ are removed under the influence of a well-defined electron beam dosage.(31) Interestingly, both works used dislocation theory to describe defects in terms of their Burgers vector, providing a link to a well-established theoretical framework and introducing these concepts to nanomaterials.

In this work, we use thermal treatments to address the relation between the crystallinity of the NCNC interfaces in a two-dimensional (2D) superlattice of epitaxially connected NCs and the optical and electronic properties of the superlattice. Although annealing has been shown to improve electronic properties in disordered films of NCs, multiple effects can arise here, one of which is a change of interparticle distance. In a NC superlattice, on the other hand, the position, the size, and the number of necks of the NCs is fixed. It therefore provides us with a model system in which we can decouple measured changes of optoelectronic properties and morphological changes of the NC array. Using elemental and structural analysis by in situ X-ray fluorescence (XRF) and grazing-incidence smallangle X-ray scattering (GISAXS), we show that such lattices keep their structural integrity up to temperatures of $\sim 300^{\circ} \mathrm{C}$. We exploit this thermal stability to assess the effect of gentle thermal annealing on the superlattice properties, where we find that annealing at temperatures ranging from $75^{\circ} \mathrm{C}$ to $150^{\circ} \mathrm{C}$ induces a marked red shift of the NC band-edge transition. In fact, it requires such an annealing step to obtain agreement between the measured band-edge transition energy and 
theoretical predictions based on tight binding calculations. In addition, we observe a 10 -fold increase of the charge carrier mobility after such mild annealing, even if such a treatment hardly effects the area of the dot-to-dot interfaces. Interestingly, while mild annealing does not change the superstructure of the NC lattice, detailed X-ray diffraction (XRD) studies point toward a marked reduction of crystalline defects upon annealing. We argue that these changes reflect the reduction of stacking faults at the interfaces connecting neighboring NCs, suggesting that the initially faulty interfaces prevent carrier delocalization over multiple NCs and restrict dot-to-dot transport of charge carriers. In this way, the results shown in this study point toward a clear strategy to promote electronic transport in NC solids by eliminating crystalline defects at NC-NC interfaces, an approach that can further enhance the performance of NC-based optoelectronic devices.

\section{Results and Discussion}

\section{Characterization of As-Prepared PbSe Nanocrystal Superlattices}

In this study, we made use of $6.5 \mathrm{~nm}$ PbSe NCs synthesized by reacting lead oleate and trioctylphosphine selenium following the approach proposed by Steckel and co-workers (see Methods section).(32) As outlined in Supporting Information S1, this synthesis method leads to monodisperse batches of quasi-spherical PbSe NCs with a first exciton transition at $0.68 \mathrm{eV}$ $(1816 \mathrm{~nm})$. Following the procedure represented in Figure 1a and described in the Methods section, $2 \mathrm{D}$ superlattices of interconnected $\mathrm{PbSe} \mathrm{NCs}$-henceforth called NC superlattices-were formed at a liquid-air interface by casting the amount of PbSe NCs needed to form a close-packed monolayer onto an ethylene glycol liquid substrate. Following a previously developed procedure,(22) the formation of a NC superlattice with square symmetry was triggered by the addition of aniline, a mild lead oleate stripping agent, to the subphase. After reacting for $30 \mathrm{~min}$, the floating NC superlattice was transferred via Langmuir-Schaeffer deposition to a Si wafer, a glass microscope slide or a transmission electron microscopy (TEM) grid.

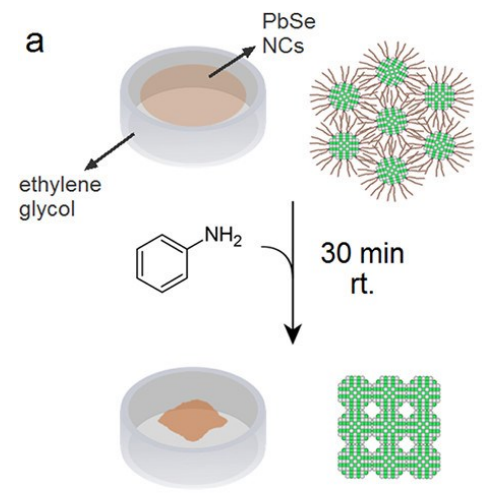

b
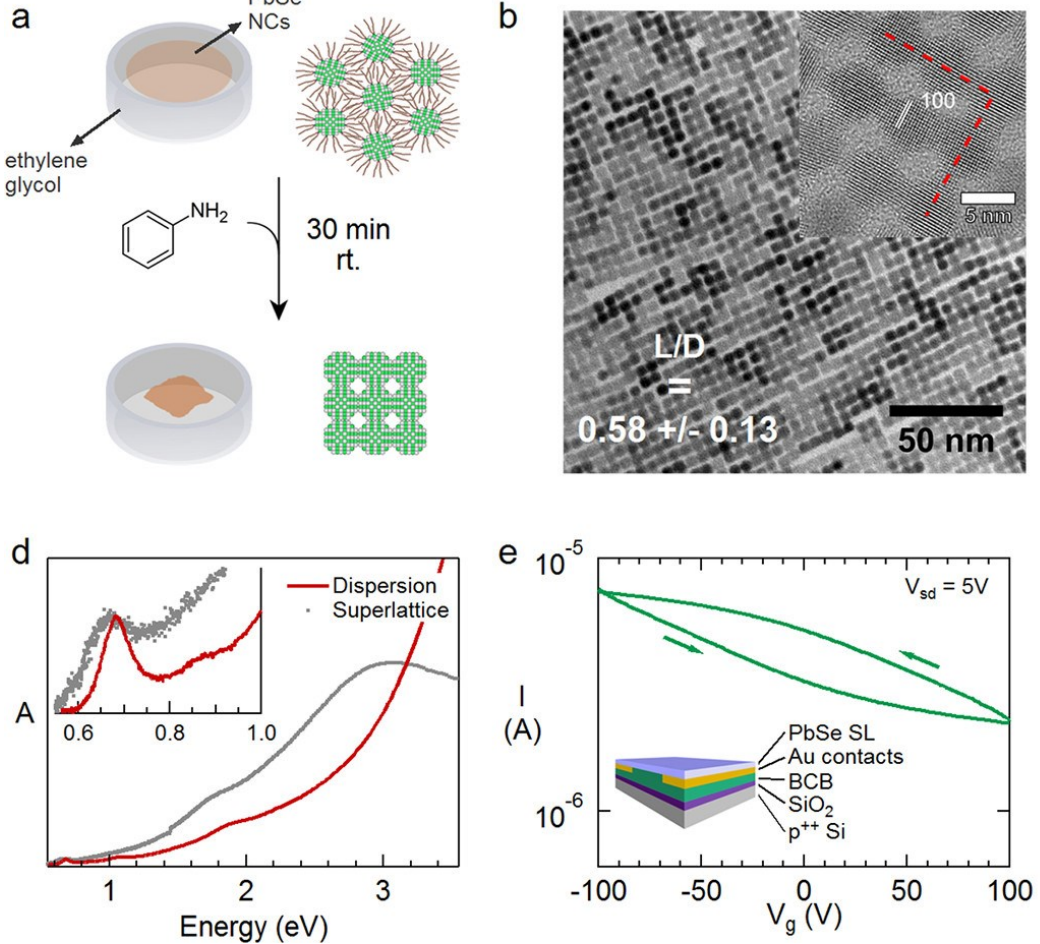
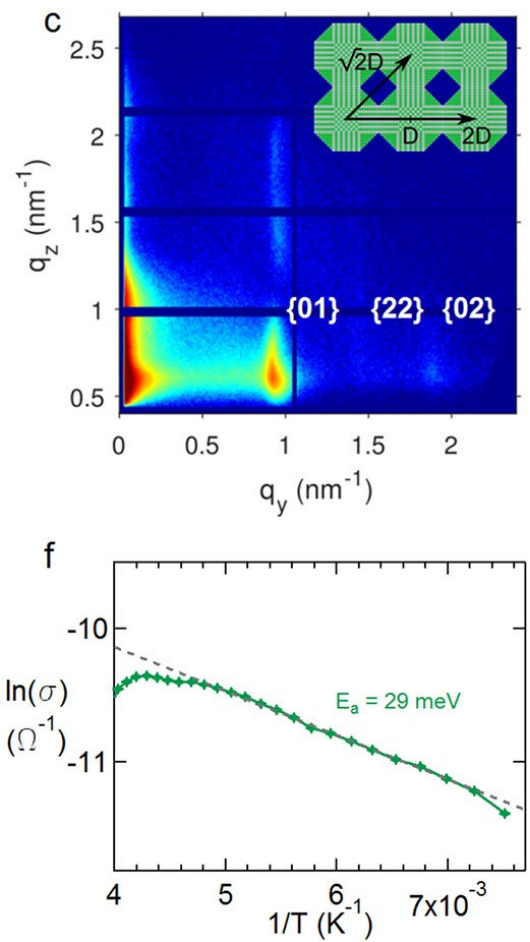

Figure 1. (a) Schematic representation of the method to synthesize NC superlattices. In this work, we use aniline as the lead-oleate stripping agent. (b) TEM image of a typical PbSe NC superlattice 
obtained through this method, showing a well-connected superlattice with an overall simple cubic symmetry. (c) A GISAXS pattern of a PbSe NC superlattice showing the $\{01\},\{22\}$, and $\{02\}$ reflections of a simple square superstructure, confirming the long-range cubic symmetry. (d) A typical absorption spectrum of an as-synthesized NC superlattice (gray) illustrating the slightly red-shifted and broadened band-edge transition compared to the colloidal dispersion (red). (e) Transfer curve of a stack of four as-synthesized NC superlattices measured on an FET device with architecture as depicted in the inset. (f) Four-probe conductivity of a stack of four NC superlattices in function of temperature.

Bright-field TEM images confirm that this procedure yields superlattices consisting of connected PbSe NCs with a simple square symmetry (see Figure 1b). By means of high-resolution TEM (HR-TEM) imaging, we can confirm that adjacent NCs are linked by apparently epitaxial interfaces formed by the connection of two (001) crystal facets. In the as-synthesized superlattice, we find an average of $3.2 \pm 0.06$ necks per NC, a number that is in line with our previous study.(22) Moreover, such HR-TEM images enable us to determine the average neck width L of these NC-NC interfaces. Normalized to the diameter $D$ of the NCs, we obtained a relative neck width L/D of $0.58 \pm 0.04$ for the structure shown in Figure $1 \mathrm{~b}$; a number that is representative for all superlattices analyzed in this study. Finally, we confirmed the long-range square symmetry of the NC superlattice through GISAXS measurements. As shown in Figure 1c, the GISAXS pattern yields reflections at scattering vectors qy of $0.96,1.45$ and $1.92 \mathrm{~nm}-1$, which can be indexed as the $\{01\},\{22\}$, and $\{02\}$ reflections of a simple square superstructure with a lattice parameter of $6.54 \mathrm{~nm}$, a number in close agreement with the NC diameter of $6.5 \mathrm{~nm}$.

Figure 1d compares the absorption spectra of a dispersion of PbSe NCs and of an as-prepared superlattice of the same PbSe NCs. At first sight, the main difference is the absorbance at around 3 $\mathrm{eV}$, which steadily increases with decreasing energy in the case of the NC dispersion but peaks in the case of the PbSe NC superlattice. This difference, however, was assigned to the reduced dielectric screening of the incident electric field by the superlattice, owing to the change of dielectric environment of the NC superlattice compared to the colloidal dispersion. This makes that the high joint density of states around the critical point in the $\Delta$ direction of the PbSe band structure leads to a peaked absorption in the case of the superlattice, not unlike the absorption spectrum of bulk PbSe.(22) More interesting for the present study is the slight red shift of the band-edge transition of the NC superlattice as compared to the NC dispersion. Such a shift was systematically observed for any superlattice analyzed throughout this investigation and typically amounted to $15-18 \mathrm{meV}$.

We evaluated charge transport in as-prepared NC superlattices by fabricating field-effect transistors using a stack of four NC superlattices as a channel connecting two cross-fingered gold electrodes. As depicted in Figure 1e, the electrodes were separated from a p++-Si gate contact by a dielectric layer consisting of $80 \mathrm{~nm} \mathrm{SiO2}$ and $180 \mathrm{~nm}$ divinyltetramethyldisiloxane-bis(benzocyclobutene) (BCB) to avoid interference from surface silanol trap states when sweeping the gate potential.(33) Each transistor consisted of 16 interdigitated electrode pairs with a length of $69 \mu \mathrm{m}$ and electrode spacing of $4 \mu \mathrm{m}$. The resulting devices always showed a transfer curve characteristic of a p-type semiconductor (see Figure 1e), which we attribute to removal of surface Pb-oleate and unintentional doping during synthesis. $(14,34)$ As described in the Methods section, we obtained a field-effect mobility from the linear part of the transfer curve, which amounted for the example shown in Figure 1 e to $2.3 \times 10-3 \mathrm{~cm} 2 / \mathrm{V} \cdot \mathrm{s}$. A low mobility in combination with the observed $p$-type conduction can originate from several factors such as, for example, partial oxidation of the NCs, something that is not uncommon for $\mathrm{Pb}$-chalcogenides. Here, the mobility is significantly smaller than what is reported in literature for NC superlattices with similar p-type behavior.(23) We attribute this difference to the 
presence of multiple cracks within the deposited superlattices and the superlattice-gold contacts (see Supporting Information S2). Since we will focus in this study on changes of the superlattice properties induced by mild thermal treatments that leave the superlattice structure unchanged, we did not attempt to further enhance the charge carrier mobility by reducing cracks or using planarized gold electrodes.

We complemented the field-effect transistor measurements by a temperature-dependent conductivity study. For this, a stack of four NC superlattices was deposited on a square of four $10 \mu \mathrm{m}$ spaced gold contacts, such that the superlattice conductance $\mathrm{G}$ could be determined from a fourprobe measurement (see Methods section). Figure $1 \mathrm{f}$ represents the thus obtained conductivity as a function of $1 / T$. This Arrhenius plot gives evidence of a thermally activated conductivity in the lowtemperature regime, which is in line with hopping transport. From the slope $d(\ln G) / d(1 / T)$, we obtain an average activation energy of $29 \mathrm{meV}$. This number is in close agreement with the hopping energy of $32 \mathrm{meV}$ reported by Whitham et al. for similar structures. As we look at transport of holes, we believe that the $29 \mathrm{meV}$ activation energy mainly reflects the variation of the upper valence band level between adjacent NCs due to the size distribution of the NC ensemble. Using the width of the band-edge absorption line as a measure for the variation of conduction and valence band levels, we can estimate the energetic distribution of the valence band levels as half the line width. From the Gaussian profile of the band edge transition, we obtain a half width at half-maximum of $35 \mathrm{meV}$, a number that is not too far from the experimental activation energy. At higher temperatures, we measure a reduction of the conductivity with increasing temperature, a characteristic property of metallic, band-like transport. However, for NC solids, it has been suggested that such a behavior can occur in activated transport when the activation energy becomes sufficiently low.(35) Since, the conductivity increases with decreasing temperature up to $\mathrm{T}=\mathrm{Ea} / \mathrm{k}$. Distinguishing between band-like transport and hopping transport based on this type of experiment is therefore not straightforward. $(35,36)$

In summary, the characterization of the as-synthesized PbSe NC superlattices confirms that the procedure used results in high-quality superlattices with long-range, square symmetry, slightly redshifted and broadened optical transitions, and transport properties where charge hopping is the main transport mechanism. Apart from the lower mobility, the aforementioned properties are in line with the properties reported in literature. We conclude that the NC solids used in this work are representative for the current state-of-the-art and are therefore a suitable starting point for further investigations.

\section{An Initial Comparison with Theoretical Expectations}

To establish a first benchmark of the properties of as-prepared PbSe NC superlattices, we compared the measured absorption spectrum of the superlattice with theoretical predictions based on tightbinding calculations (see Methods section for details). Previously, such calculations showed that in the case of PbSe NC superlattices, the eight-fold degeneracy of the valence- and conduction bandedge states of the individual PbSe NCs yields a superlattice with a rich electronic structure that depends on the symmetry of the assembly, on the size and shape of the NCs and on the coupling of adjacent NCs.(26-28) Here, we calculated the electronic band structure as a function of the relative width of the necks connected neighboring NCs for the square superlattice of PbSe NCs shown in Figure 2a, where an increased neck width results in larger coupling energies Vss $\sigma$ and Vppo between the $1 \mathrm{~S}$ and $1 \mathrm{P}$ states of adjacent NCs. Figure $2 \mathrm{~b}$ represents the band structure obtained for a square lattice of $5.5 \mathrm{~nm}$ PbSe NCs with a relative neck width of 0.1 and 0.6 , respectively. In the former case, 
the small interdot coupling leads to narrow energy bands that essentially coincide with the different quantized states of isolated PbSe NCs. Increasing the relative neck width to 0.6 , on the other hand, results in strongly dispersive energy bands. This makes that such a superlattice will have a significantly smaller band gap than the isolated PbSe NCs.

a

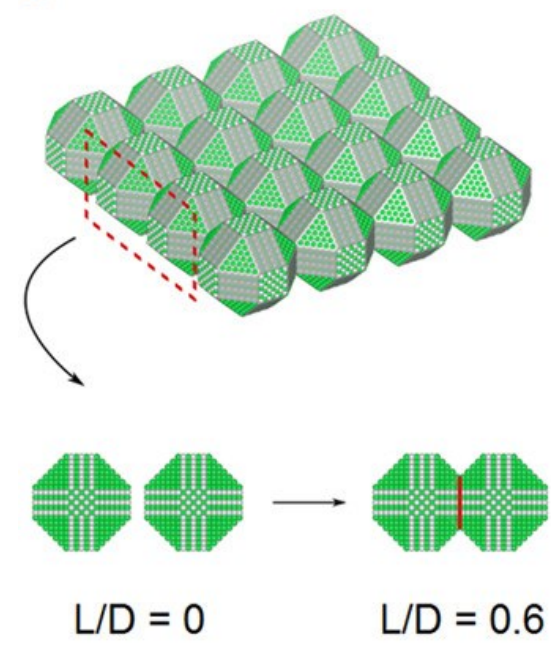

b
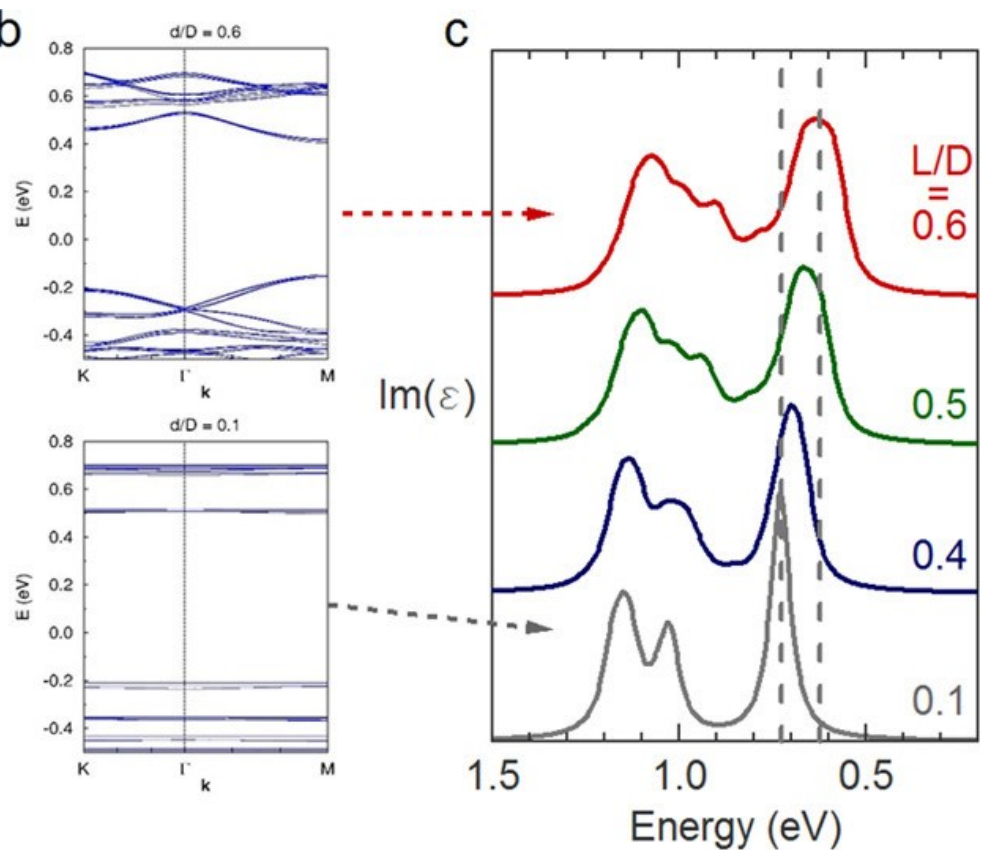

Figure 2. (a) Schematic representation of the model used in the tight binding calculations. The neck width $\mathrm{L}$, the $2 \mathrm{D}$ projection of the epitaxial bond, is indicated by the red line. (b) The calculated electronic structures for superlattices with an L/D ratio of 0.6 (top) and 0.1 (bottom). For a low L/D ratio, the bands coincide with the energy levels of the individual NCs. At an increasing L/D ratio, dispersive bands start to form. (c) The dielectric function of the superlattice, calculated from the theoretical band structure. For $L / D=0.6$, the first excitonic transition red shifts by $99 \mathrm{meV}$ and the fwhm increases by a factor 2.5 compared to the superlattice with $L / D=0.1$.

To link these calculations to the experimental characteristics of the PbSe superlattice presented in Figure 1, we calculated the imaginary part $\epsilon l$ of the dielectric function of the superlattice from the theoretical band structure for different relative neck widths. While this approach involves a single particle approximation that neglects excitonic effects, we use this spectrum of $\epsilon l$ as a first indication of the band-edge shift to be expected in PbSe NC superlattices. As shown in Figure $2 \mathrm{c}, \in \mathrm{El}$ exhibits the narrow absorption features of isolated PbSe NCs in the case of a superlattice with a relative neck width of 0.1 . At $0.73 \mathrm{eV}(1700 \mathrm{~nm})$, the band-edge transition of this superlattice is only shifted by $\sim 25 \mathrm{meV}$ to the red as compared to the isolated NCs. At a relative neck width of 0.6 , on the other hand, the dispersive energy bands lead to a further shift of the band-edge transition by $99 \mathrm{meV}$ and an increase of the full-width-at-half-maximum of the band-edge feature to $97 \mathrm{meV}$, as compared to a mere $38 \mathrm{meV}$ for the structure with a relative neck width of 0.1 . We thus conclude that the coupling between adjacent NCs that comes with a relative neck width of 0.6 should have a substantial effect on the optical properties of the superlattice, in particular near the band-edge transition; an effect that should be readily measurable through absorption spectroscopy. Furthermore, we expect that the optical properties of the superlattice are very sensitive to small changes in the coupling strength once the relative neck width exceeds 0.4 , where we find that increases of $L / D$ by 0.1 lead to successive red shifts of the band-edge transition by more than $30 \mathrm{meV}$. 
As compared to the theoretical predictions summarized in Figure 2, the as-prepared NC superlattices show a very small band-edge shift. While TEM analysis yields a relative neck width close to the value of 0.6 used in the calculations, only a modest band-edge shift by $18 \mathrm{meV}$ is measured. A relative neck width of 0.6 is comparable to what Whitham et al. reported(24) and is slightly smaller than the value of 0.69 reported by Evers et al. and Alimoradi Jazi et al. for superlattices fabricated at more elevated temperatures.(21,37) Even so, the latter authors also reported relatively small band-edge shifts of about $25 \mathrm{meV}$. This discrepancy between experiment and theory indicates that either the theoretical model used does not accurately predict the optical properties of PbSe NC superlattices or that the current method of producing NC superlattices does not give the anticipated electronic coupling between NCs, even if the relative neck widths suggest otherwise. In this respect, it is important to realize that the premise of our theoretical approach is that adjacent NCs are connected through defect-free, epitaxial necks that lead to a perfect relative alignment of the NCs. Given the fact that $\mathrm{PbSe} \mathrm{NC}$ superlattices are prepared at room temperature, the structure of real necks might be very different from such an idealized case and contain defects, vacancies or dislocations. Supporting Information S3 illustrates several of such defects at the NC-NC interface, which originate from slight misalignments between the NCs. The presence of such faulty interfaces might be one reason why the absorption spectrum of a PbSe NC superlattice fails to reproduce the band gap reduction that epitaxial inter NC connections should bring about.

\section{Thermal Annealing of PbSe Nanocrystal Superlattices}

Thermal annealing is a well-known strategy to induce grain growth in a polycrystalline material, where thermal activation enables atoms to migrate and eradicate dislocations. Looking at the NC-NC interfaces in a NC superlattice as grain boundaries, we therefore attempted to anneal NC superlattices to remove possible dislocations and defects at the NC-NC interface, without affecting the overall structure of the superlattice. To evaluate the stability of connected PbSe NC superlattices under thermal annealing, we tracked changes in the structure and the elemental composition of a given NC superlattice by in situ GISAXS and XRF. As detailed in the Methods section, these measurements made use of a sample chamber mounted in the path of a $14 \mathrm{keV} \mathrm{X-ray} \mathrm{beam} \mathrm{at} \mathrm{the}$ DUBBLE BM26B beamline of the European Synchrotron Radiation Facility (ESRF, Grenoble, France). The sample chamber was equipped with a dedicated heating stage and Be windows to enable in situ GISAXS and XRF during thermal annealing in He atmosphere.

In a first series of experiments, we annealed PbSe NC superlattices in He up to $375^{\circ} \mathrm{C}$ at a heating rate of $0.2^{\circ} \mathrm{C} / \mathrm{s}$. Figure 3 a shows a selection of GISAXS patterns of a given superlattice at $75^{\circ} \mathrm{C}, 200$ and $375^{\circ} \mathrm{C}$. The $75^{\circ} \mathrm{C}$ GISAXS pattern exhibited a main diffraction spot at a scattering vector qy of $0.96 \mathrm{~nm}-1$ and the second order signal at $1.92 \mathrm{~nm}-1$, which correspond to a NC spacing of $6.54 \mathrm{~nm}$. Similar features were indexed as the $\{01\}$ and $\{02\}$ reflections in the GISAXS pattern of an as-prepared square superlattice with a lattice parameter of $6.5 \mathrm{~nm}$, see Figure 1c. Increasing the temperature up to $200^{\circ} \mathrm{C}$ did not noticeably change this GISAXS pattern, which indicates that both the in-plane square symmetry and the NC-NC spacing are preserved. On the other hand, all diffraction features of the superlattice disappeared at a temperature of $375^{\circ} \mathrm{C}$. A more detailed analysis showed that the intensity of the $\{01\}$ diffraction feature remains steady up to a temperature of $300^{\circ} \mathrm{C}$, see the green trace in Figure $3 \mathrm{~b}$. Similarly, the elemental composition of the PbSe superlattice is unchanged, with a $\mathrm{Se}: \mathrm{Pb}$ signal ratio slightly larger than 0.8 . Increasing the temperature further, however, resulted in a rapid loss of the diffraction features and a pronounced drop of the Se:Pb signal ratio. As detailed in Supporting Information S4, a similar transition temperature was found with $5.1 \mathrm{~nm}$ PbSe NCs and PbSe NCs with a different surface termination. 

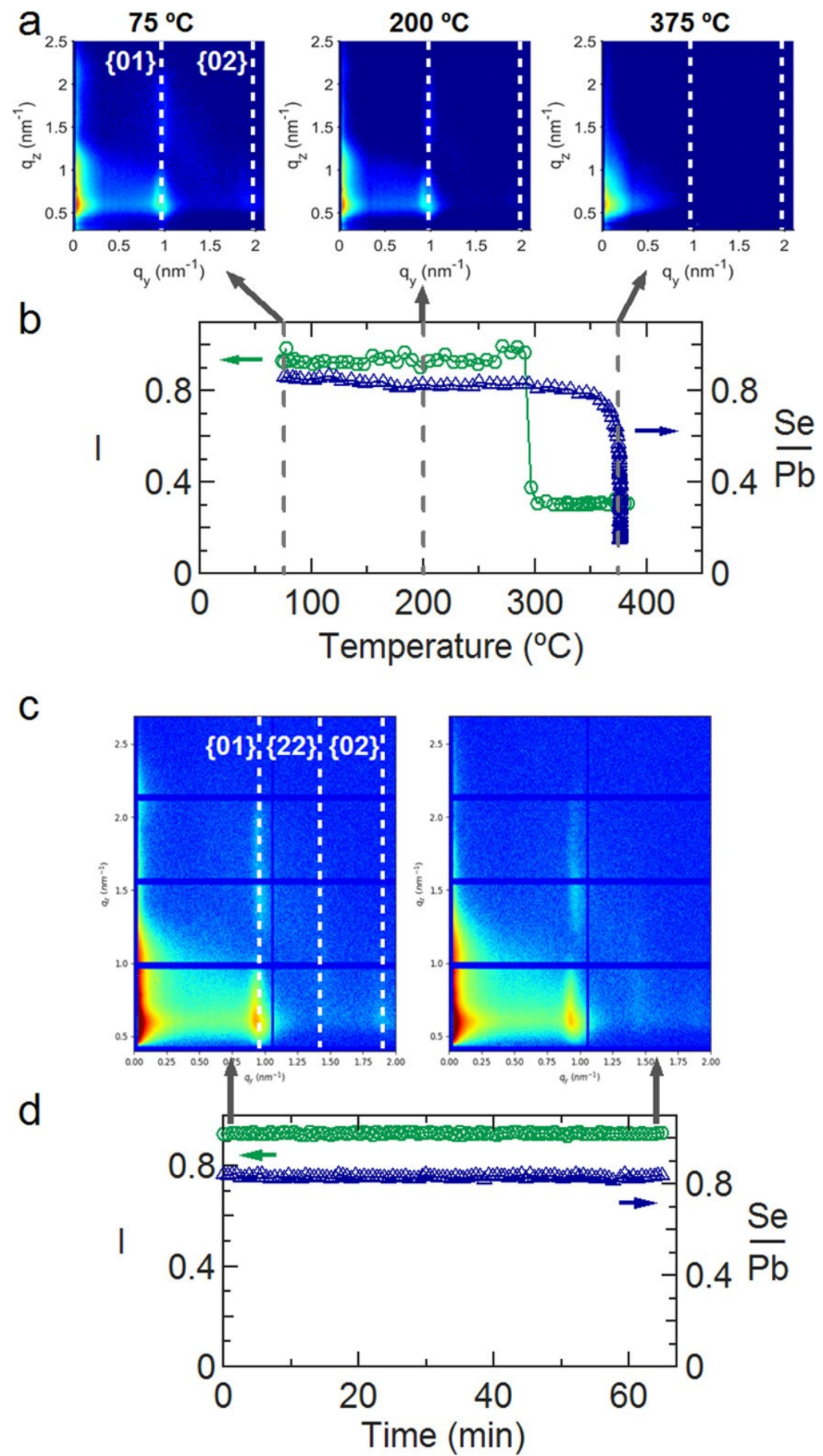

Figure 3. (a) GISAXS patterns of the PbSe NC superlattice at the indicated temperatures during the in situ annealing experiments. (b) Evolution of (green) the intensity of the $\{01\}$ reflection and (blue) the $\mathrm{Se}: \mathrm{Pb}$ molar ratio as a function of temperature. It can be seen that the superlattice reflections disappear at $\sim 300^{\circ} \mathrm{C}$, while the Se:Pb XRF ratio drops at temperatures above $350{ }^{\circ} \mathrm{C}$. (c) GISAXS patterns of a PbSe NC superlattice before and after annealing for $1 \mathrm{~h}$ at $150^{\circ} \mathrm{C}$. (d) Evolution of (green) the intensity of the $\{01\}$ superlattice reflection and (blue) the Se:Pb ratio while keeping the superlattice at $150^{\circ} \mathrm{C}$. Both signals remain steady during this low-temperature anneal.

In Figure 3c, we present the results of an experiment in which a connected PbSe NC superlattice is annealed for more than $1 \mathrm{~h}$ at a temperature of $150^{\circ} \mathrm{C}$, that is, well within the thermal stability 
window. Clearly, the superlattice gives rise to identical GISAXS patterns at the start and the end of the annealing process, and the elemental composition of the superlattice remains constant, see Figure $3 \mathrm{~d}$. Moreover, a combined thermal gravimetric/differential thermal analysis (TGA/DTA) indicates that the degradation of the lead oleate capping of the PbSe NCs used here does not occur below temperatures of $235^{\circ} \mathrm{C}$ (see Supporting Information S5). We thus conclude that the overall superlattice structure, the NC-NC spacing, and the NC composition can be preserved for a relatively long time under mild annealing conditions, characterized by an upper temperature of $\sim 300^{\circ} \mathrm{C}$ for structural changes. This finding enables thermal routes of defect removal in NC solids, without compromising the superlattice structure but also sets limits to the thermal budget during manufacturing of NC-based devices. As a final remark, we point out that this thermal stability study involved dry superlattices deposited on Si wafers. Superlattices on the surface of an ethylene glycol liquid subphase are far less stable. As shown in Supporting Information S6, annealing at a mere $50^{\circ} \mathrm{C}$ can suffice to destroy the superlattice under such conditions, yielding a structure that closely resembles a network of molten NCs.

\section{Optical and Electronic Properties of Annealed Superlattices}

Figure 4a represents absorption spectra of PbSe NC superlattices annealed at different temperatures as indicated. While all these annealing temperatures fall well within the established thermal stability window, one sees that annealing induces pronounced changes in the absorption spectrum. In particular, the feature of the band-edge transition exhibits a marked broadening and a pronounced shift to longer wavelengths with increasing annealing temperature. At an annealing temperature of $150^{\circ} \mathrm{C}$, for example, we retrieve a red shift of about $70 \mathrm{meV}$ as compared to the as-prepared superlattice. Clearly, such a shift and broadening of the band-edge transition agrees much better with the shift predicted through tight-binding calculations, see Figure $2 \mathrm{c}$. Additionally, we find that the optical transition at around $3 \mathrm{eV}$ decreases in energy, which is in agreement with a gradual shift toward the bulk spectrum of PbSe (see Supporting Information S7).

\section{a}

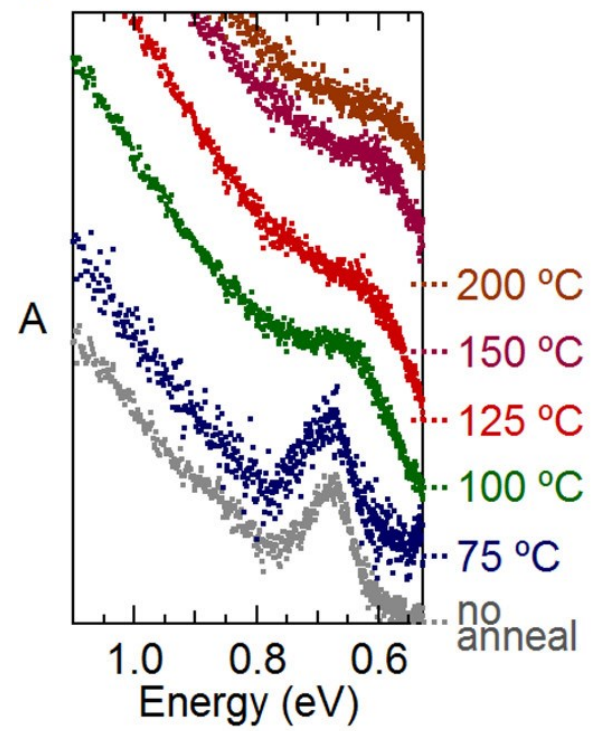

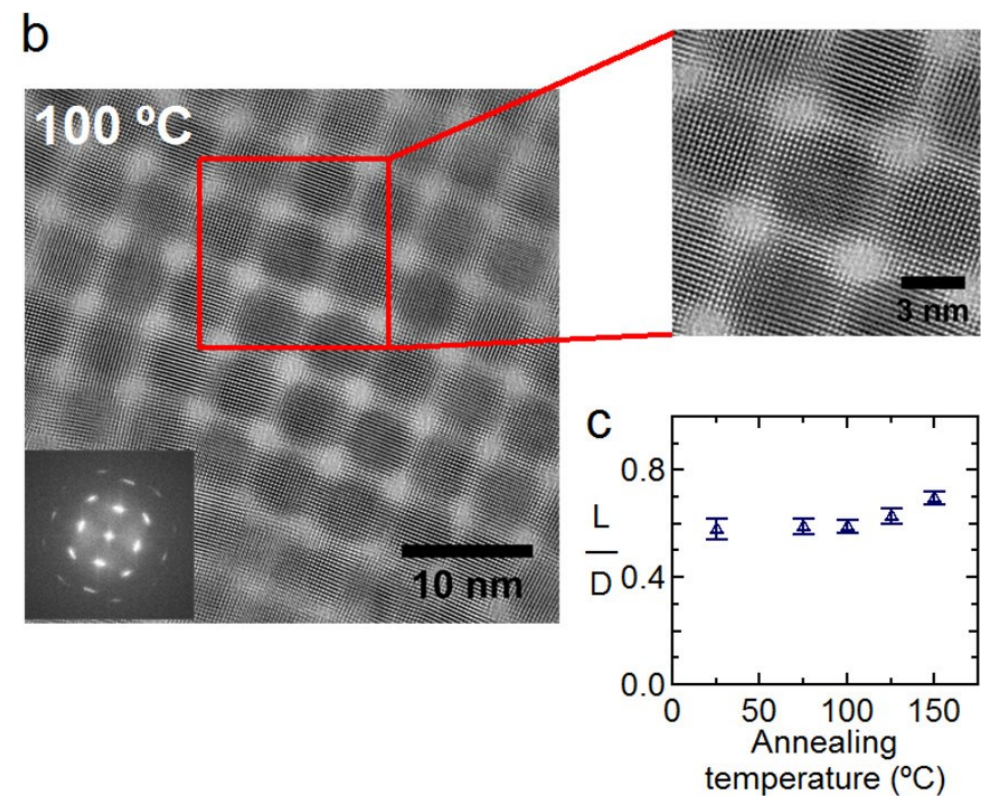

Figure 4. (a) Optical absorption spectra of PbSe NC superlattices annealed for $1 \mathrm{~h}$ at the indicated temperatures. The spectra are normalized to the absorption peak of the superlattice before annealing. (b) HR-TEM image of a PbSe NC superlattice annealed for $1 \mathrm{~h}$ at $100^{\circ} \mathrm{C}$ showing that the NC shape is preserved after annealing. The inset shows the Fourier transform of the image. The 
enlarged region illustrates the quality of the epitaxial connections after annealing. (c) Analysis of the $L / D$ ratio determined from HR-TEM images for several annealing temperatures. The NC diameter is found to be constant at 5.9-6.0 $\mathrm{nm}$ throughout the annealing series. The L/D ratio increases from 0.58 without annealing to 0.70 after annealing for $1 \mathrm{~h}$ at $150^{\circ} \mathrm{C}$.

In order to properly interpret the changes in the absorption spectrum brought about by mild thermal annealing, we examined the superlattices after heat treatment by TEM. As shown in Supporting Information S4, low-magnification TEM and electron diffraction patterns confirm that the annealed superlattices preserve the long-range order and the square symmetry of the as-prepared superlattices, observations that complement the conclusions we obtained from GISAXS measurements. Furthermore, HR-TEM images (see Figure $4 \mathrm{~b}$ ) indicate that the NC shape is preserved upon annealing, with the average diameter staying constant at 5.9-6.0 nm throughout the annealing series. Here, the diameter is measured as the sphere circumfering the (011) planes, due to the difficulty in accurately determining the NC edge across the (001) necks. This method gives consistent results, but induces a slight offset compared to the center-to-center distance observed through GISAXS. An important parameter characterizing the connectivity of the NC superlattice is the number of necks per NC. From HR-TEM images, we found that this neck number varies around 3 necks per $\mathrm{NC}$, irrespective of the annealing temperature (see Supporting Information S4 for a quantitative analysis). In agreement with our previous analysis of NC superlattices,(22) this result indicates that no new necks are formed during annealing. Finally, we estimated the average relative neck width L/D as a function of temperature. As shown in Figure 4c, we find that the relative neck width stays constant at 0.59 for annealing temperatures up to $100^{\circ} \mathrm{C}$ and slightly increases to $0.70 \pm 0.02$ at $150^{\circ} \mathrm{C}$ (see Supporting Information S4 for a quantitative analysis of the relative neck width and the amount of necks per NC). These figures make the superlattice annealed at $100^{\circ} \mathrm{C}$ particularly interesting since this sample already exhibits a marked red shift and broadening of the band-edge absorption, without annealing inducing changes to the superlattice structure, the NC diameter, nor the relative neck width.

The combination of the optical absorption spectra and the TEM measurements on annealed superlattices indicates that a thermal treatment relaxes charge carrier confinement, without changing the size and shape of the NC building blocks. This points toward an increased delocalization of the electronic states in the NC solid, leading to a band-edge shift in better agreement with theoretical predictions. Importantly, we found that this increased delocalization is not the mere result of an increased neck width as measured from TEM, since we already observe relaxation of confinement without significant changes of the relative neck width, for example, in the sample annealed at $100{ }^{\circ} \mathrm{C}$. This indicates that the electronic coupling between adjacent NCs is not a mere function of the relative neck width, but may also be affected by, for example, defects at the NC-NC interface that are eliminated by thermal annealing. From this perspective, the optical absorption spectrum can be seen as probing an effective neck width, which can be defined as the width of a defect-free neck that would yield the observed band-edge shift.

Extending our reasoning from optical to electronic properties, one expects that improved charge carrier delocalization also enhances the charge carrier mobility. To test this conjecture, we annealed a field-effect transistor made of four stacked PbSe NC superlattices (see Figure 1e) for $1 \mathrm{~h}$ at $150^{\circ} \mathrm{C}$ and compared the mobility before and after annealing. Figure 5 a shows the transfer curve of this annealed transistor, from which we obtain a field-effect mobility of $1.9 \times 10-2 \mathrm{~cm} 2 / \mathrm{V} \cdot \mathrm{s}$, that is, an almost 10 -fold enhancement. The I-V curves of this device are shown in Supporting Information 58 . Intriguingly, a similar analysis on monolayer superlattices yields mobility enhancements by a factor of 
1000 , see Supporting Information S8. Similarly, annealing of a stack of four NC superlattices at a temperature of $100^{\circ} \mathrm{C}$ results in an enhancement of the mobility by a factor of $3.7 \pm 0.3$ (see Supporting Information S8). Figure $5 \mathrm{~b}$ plots the differential mobility as a function of the amount of injected charges in the NCs (see Methods section). From the gate capacitance and assuming a surface density of NCs of $2.31012 \mathrm{~cm}-2$ in the square lattice, we estimate that the gate voltage sweep from +100 to $-100 \mathrm{~V}$ adds 5 holes per NC. We find that this leads to an increase of the differential mobility at first, which peaks at a charge density of $n i+2$, and decreases to a minimum at $n i+5$, where $n i$ is the carrier density at $+100 \mathrm{~V}$. A similar variation of the differential mobility was observed during the gradual filling of $\mathrm{S}$ and $\mathrm{P}$ bands in $\mathrm{CdSe}$ and $\mathrm{HgSe} \mathrm{NC}$ films, where the differential mobility reaches a maximum around a half-filled band. $(38,39)$ In the case of PbSe, the eight-fold degeneracy of the $S$ band would result in a maximum mobility at 4 charges per NC, which is in reasonable agreement with the data in Figure $5 b$, considering that charges are already present at the initial gate voltage of +100 $\mathrm{V}$. This suggests that we measure carrier transport through the NC band-edge states, and not through a series of impurity-related trap states. In this case, the rather low mobility reflects the percolative pathway between the electrodes caused by cracks and poor contacts, yet since annealing leaves this superstructure unchanged, the relative change of the mobility upon annealing still reflects intrinsic changes in the superlattice, for example, due to an improved NC-NC coupling. Together with the increasing mobility, we also find that the average activation energy for charge transport is lowered. Looking at the four-probe conductivity after annealing in Figure 5c, the initial activation energy of 29 meV reduces to $13 \mathrm{meV}$ after $1 \mathrm{~h}$ at $150^{\circ} \mathrm{C}$. This experiment shows that by annealing, the average energy barrier, which is a measure for the spread of the distribution of energy levels, is reduced, thereby effectively homogenizing the energy landscape of the superlattice.
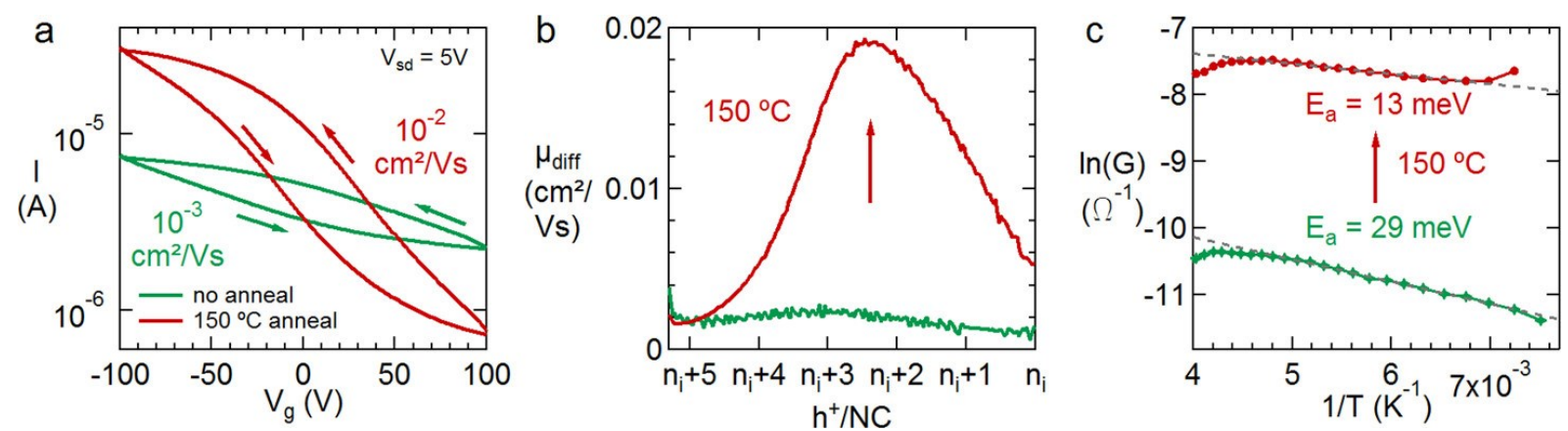

Figure 5. (a) Transfer curves of a stack of four NC superlattices before and after annealing for $1 \mathrm{~h}$ at $150{ }^{\circ} \mathrm{C}$. The extracted mobility shows a $\sim 10$-fold enhancement after annealing. (b) The differential mobility as a function of the number of injected holes into the NC superlattice, with ni the carrier density at $\mathrm{Vg}=+100 \mathrm{~V}$. The behavior of the differential mobility reflects a filling of the eight-fold degenerate $S$ band. (c) Four-probe conductivity of a stack of 4 NC superlattices in function of temperature. Annealing reduces the activation energy from 29 to $13 \mathrm{meV}$.

\section{Structural Changes in Annealed Superlattices}

The combination of structural analysis by GISAXS and TEM and optical and electrical characterization of annealed $\mathrm{PbSe} \mathrm{NC}$ superlattices indicates that a thermal treatment improves the electronic coupling between adjacent NCs, without necessarily increasing the relative neck width of the NC-NC interface. To address the conjecture that annealing eliminates defects or dislocations at the NC-NC interface, a process not uncommon in polycrystalline bulk materials, we sought to investigate in more detail the crystallinity of PbSe NC superlattices before and after annealing. An interesting observation from this perspective is that the (002) diffraction feature of a square superlattice of $\mathrm{PbSe}$ 
NCs can be recorded using a regular powder diffractometer since the PbSe NCs in such a superlattice have their (001) facets aligned parallel to the substrate. This makes that the distribution of out-ofplane orientations or the mosaicity of a PbSe NC superlattice can be measured by setting the diffraction angle at the Bragg condition for the (002) planes of PbSe and rocking the substrate over an azimuthal angle $\Omega$. As shown in Figure $6 a$, the thus obtained rocking curve will feature a narrow line centered at $\Omega=0$ for an ensemble of perfectly aligned NCs, whereas broader rocking curves will be obtained on ensembles with a larger mosaicity.

a
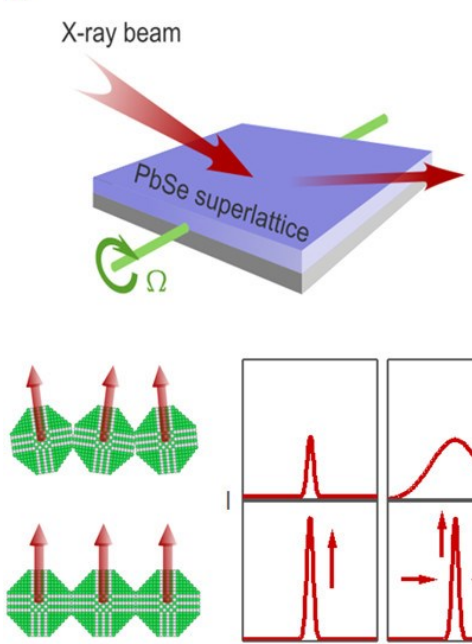

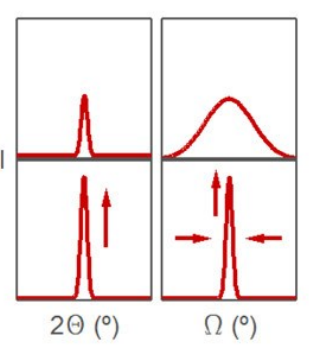

$\mathrm{b}$
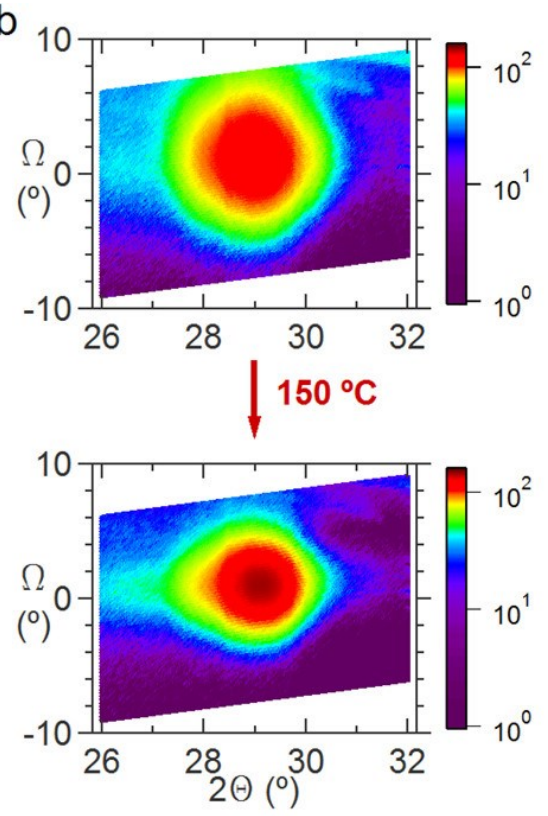

C

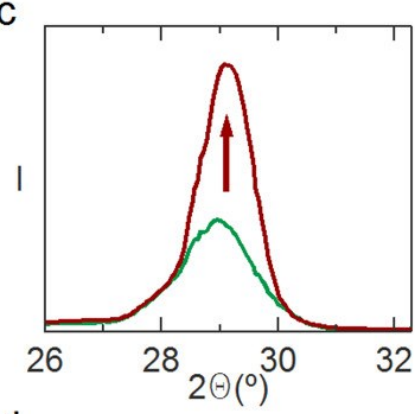

d

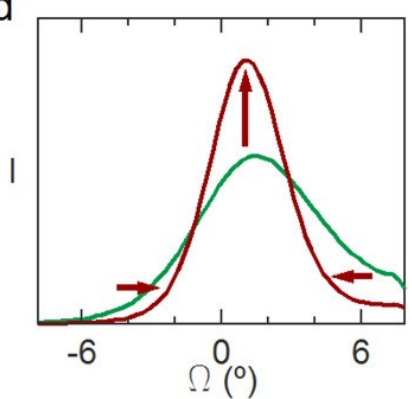

Figure 6. (a) Schematic representation of the rocking curve XRD measurement. An additional rotation angle $\Omega$ is introduced to evaluate the mosaicity of the NC superlattice. A broad signal in the Ivs $\Omega$ curve represents a high mosaic spread with a large distribution of surface normals in the probed reciprocal space, while a sharp signal represents a narrow distribution of surface normals. (b) Reciprocal space maps of the PbSe (002) peak of the NC superlattice before and after annealing for 1 $\mathrm{h}$ at $150^{\circ} \mathrm{C}$. The peak intensity increases, and the width in the direction of $\Omega$ is reduced after annealing. (c) Horizontal profile of the reciprocal space maps in (b), plotting the diffraction intensity integrated from $\Omega=-2^{\circ}$ to $\Omega=+2^{\circ}$ for every angle $\theta$. (d) Vertical profile of the reciprocal space maps in (b), plotting the diffraction intensity integrated from $\theta=26^{\circ}$ to $\theta=32^{\circ}$ for every angle $\Omega$. Annealing for $1 \mathrm{~h}$ at $150^{\circ} \mathrm{C}$ reduces the mosaicity of the NC superlattice, as illustrated by the increased population of $\mathrm{NC}$ aligned to the substrate and the reduced population of NC with larger misalignment angles.

Figure $6 \mathrm{~b}$ represents a map of the $\mathrm{X}$-ray intensity diffracted by a given PbSe NC superlattice as a function of $\theta$ and $\Omega$, before and after a $1 \mathrm{~h}$ anneal at $150^{\circ} \mathrm{C}$. In both cases, the (002) diffraction feature of the superlattice can be clearly discerned, and it appears that annealing leads to a more intense diffraction spot that is narrowed down along $\Omega$. These observations are confirmed by Figure $6 c, d$, which represent the diffraction intensity integrated from $\Omega=-2^{\circ}$ to $\Omega=+2^{\circ}$ for every angle $\theta$ and from $\theta=26^{\circ}$ to $\theta=32^{\circ}$ for every angle $\Omega$, respectively. As can be seen in Figure $6 c$, annealing results in a significant increase of the crystalline volume within the $\pm 2^{\circ}$ range of azimuthal angles. Following Figure $6 \mathrm{~d}$, this increase comes at the expense of the crystalline volume with a larger outof-plane orientation. We thus conclude that annealing effectively reduces the mosaicity of a given NC 
superlattice and leads to a more homogeneous overall alignment of the superlattice with the substrate.

A quantitative analysis of the disorder in PbSe NC superlattices arises from investigating the widths of a series of Bragg peaks. An inhomogeneous crystallographic disorder caused by vacancies, interstitials, dislocations, and mosaicity results in local distortions of the lattice parameter and lattice angle. Following Bragg's law, this translates into a wider spread of diffracted beams in both directions parallel and perpendicular to the reciprocal space vector q, defined here as the (001) direction perpendicular to the substrate. The recorded XRD pattern, therefore, shows broader features compared to a crystal without the disorder. In the case of NCs, the inverse crystallite size also makes diffraction peaks broader, which makes the recorded diffraction peak width a combination of size and disorder broadening. Since both effects have a different dependency on the diffraction angle, their contributions to the peak width can be separated by recording higher order diffraction peaks of the same lattice planes, known as the Williamson-Hall method. While the disorder broadening in the direction parallel to $q$ arises due to strain gradients (microstrain), that is, a broadened distribution of lattice parameters, the disorder broadening in the direction perpendicular to q stems from mosaicity, that is, the angular misalignment of crystal planes in the out-of-plane direction (see Figure 6a).(40)

We recorded the (002), (004), and (006) out-of-plane X-ray diffraction peaks of pristine and ex situ annealed PbSe NC superlattices by using the Cornell High Energy Synchrotron Source (CHESS). By using a 2D detector, we collected three-dimensional reciprocal space maps and analyzed the width of the diffraction peak both in the direction parallel and perpendicular to the (001) direction in reciprocal space. As expected, there is no difference between the directions perpendicular to (001) due to the random in-plane orientation of the NC superlattices. Peak fitting revealed Gaussian peak profiles in both (001) and (100) directions. Following the Williamson-Hall analysis for Gaussian peak profiles, the dependency of the squared peak width $(\delta q)^{2}$ on $q^{2}$ follows a linear model, allowing the separation of disorder (slope of the linear model) and size (the inverse intercept at $q=0)$. (40) Figure 7 a shows the squared widths $(\delta q)^{2}$ as a function of $q^{2}$ for ex situ annealed PbSe NC superlattices in the direction parallel to $\mathrm{q}$. The microstrain determined from the slope of the linear model (see Figure 7b) monotonically drops as a function of annealing temperature from 0.027 in the as-synthesized NC superlattice to 0.017 after annealing at $150{ }^{\circ} \mathrm{C}$. The microstrain has been attributed to small-scale variations due to point defects $(41)$ and dislocations; $(40,42)$ however, determining the defect density from the microstrain is challenging.(40) We hypothesize strain gradients at the boundary between the NCs, which rearrange into a lower-strain energy configuration during annealing at high temperatures. The intercept $\delta q_{0}$ of the linear model (see Figure $7 \mathrm{c}$ ) determines the crystallite size through $d=2 \pi / \sqrt{\delta q_{0}}$. From the data parallel to the q direction, we estimate a crystallite size of 6.6 $\pm 0.4 \mathrm{~nm}$ and observe a weak dependence on temperature, in agreement with our previous TEM and GISAXS measurements. 


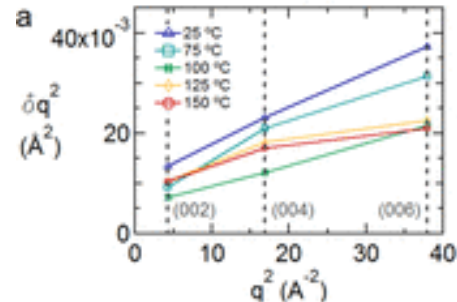

d

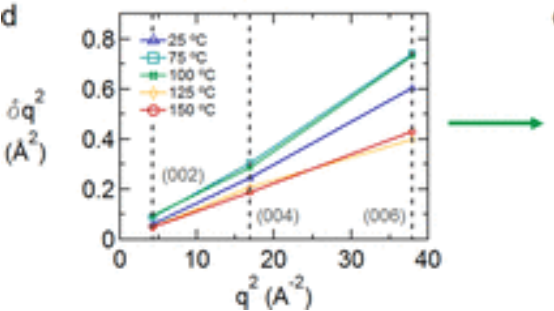

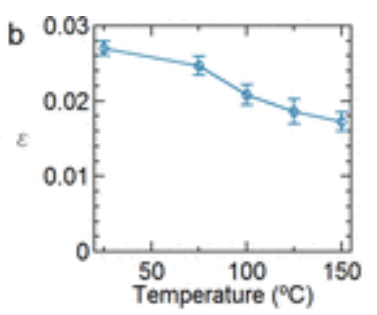

e

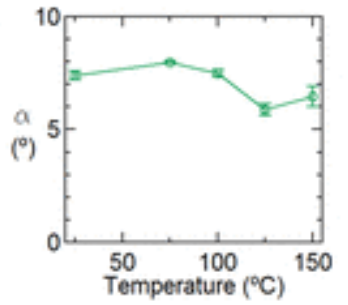

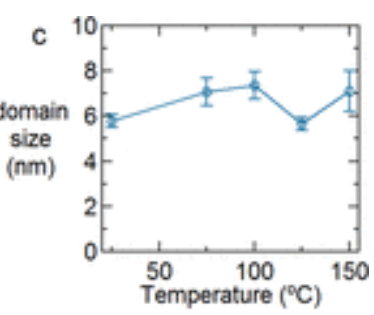

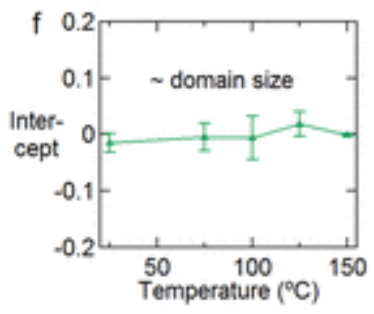

Figure 7. Size-strain analysis through the Williamson-Hall method. (a) Plots of $(\delta q)^{2}$ parallel to the $q$ direction in function of $q^{2}$ for the (002), (004), and (006) diffraction peaks of NC superlattices annealed at the indicated temperatures. The q direction is defined as (001) (perpendicular to the substrate). (b, c) Analysis of the microstrain $(\varepsilon)$ and domain size in the NC superlattices. The slope of the linear fits to the data in (a) scales with the microstrain, while the intercept inversely scales with the domain size. (d) Same as (a) for $(\delta q)^{2}$ perpendicular to the q direction. (e) Analysis of the mosaicity $(\alpha)$ in the NC superlattice. The mosaicity scales with the slope of the plots in (d). (e) The intercept of the linear fits to the plots in (d), which scales with the domain size. A number for the domain size could not be extracted here, owing to the negative values of the intercept.

The Williamson-Hall analysis in the direction perpendicular to q corroborates the reduction of the disorder. The slope determined from the linear model of peak widths shown in Figure $7 \mathrm{~d}$ reveals an improved alignment of crystal planes upon annealing: The width of the angular distribution $\alpha$ of (001) planes reduces from $7.3^{\circ}$ before annealing to $6.1^{\circ}$ after annealing at $150{ }^{\circ} \mathrm{C}$ (see Figure 7e), in agreement with the rocking curve data in Figure 6. Intuitively, the mutual alignment of neighboring NCs results in a reduction of the dislocation density concentrated at the interfaces between NCs. In analogy to low-angle boundaries in macroscopic crystals, we estimate the dislocation density from $D$ $=\alpha / 2 b d$, where $b$ is the Burgers vector and $d$ is the average crystal size.(43) Considering $d=6.5 \mathrm{~nm}$ and taking a Burgers vector component perpendicular to the substrate of $b=3.06 \AA$, we estimate that the dislocation density decreases from $3.2 \times 1012 \mathrm{~cm}-2$ to $2.6 \times 1012 \mathrm{~cm}-2$ after annealing, which represents a reduction from $\sim 1$ defect per 2 NCs to $\sim 1$ defect per 3 NCs. The choice of Burgers vector is determined by the fact that only the out-of-plane Bragg peak is recorded in this measurement, meaning that this measurement is only sensitive toward the out-of-plane component of the Burgers vector. It has been shown by Ondry et al. that the in-plane dislocations in epitaxially connected NCs have a Burgers vector of $b=\frac{a}{2}[011]$.(31) Assuming similar dislocations, the perpendicular component of this vector along the [001] direction becomes $b=a / 2=3.06 \AA$, which we use in our calculation of the defect density. The intercept $\delta q_{0}$ for the in-plane direction (see Figure $7 f$ ) is much smaller than the intercept in the out-of-plane direction; the crystal size in-plane is defined by the size of the mesocrystal containing multiple NCs. For large crystals, the analysis of the intercept becomes sensitive to the exact functional form of the peaks, which may deviate from a Gaussian form assumed here. Therefore, we do not have sufficient precision to differentiate between domain sizes at different temperatures.

\section{Theoretical Insights on Carrier Localization through Defective Interfaces}


The experiments presented here show that despite long-range order on the superstructure level of as-synthesized NC superlattices, a substantial amount of disorder remains at the atomic level. The disorder is experimentally found to originate from NC mosaicity, which suggests that adjacent NCs connect through small-angle grain boundaries or, concomittantly, two facets joined together by an array of edge dislocations, which reduces the grain boundary to a series of point defects. To evaluate the effect of such defective interfaces on electronic coupling, we analyzed the electronic structure of a PbSe dimer, consisting of two epitaxially connected PbSe NCs. As illustrated in Figure 8a, each NC can be seen as an artificial atom characterized by one $S$ orbital in the valence band and one in the conduction band.(29) Upon bonding, the linear combination of the NC orbitals results in a bonding dimer orbital with lower energy and an antibonding dimer orbital with a higher energy. The coupling energy $2 \mathrm{Vss} \sigma$ represents the strength of the bond between the neighboring NCs and is denoted as the splitting energy between the bonding and antibonding dimer orbitals in Figure 8a. In reality, the $\mathrm{PbSe}$ system is more complex because of valley degeneracy, and the splitting energy is therefore calculated as the average over the eight bonding and eight antibonding states. Since the NC S orbitals are a product of the atomic states that make up the NC, changes to the atomic configuration will affect the NC S orbital and thus the splitting energy in the dimer molecule. This allows us to introduce some forms of disorder and evaluate its effect on the electronic coupling. Figure $8 \mathrm{~b}$ shows the thus obtained splitting energies for a NC dimer where increasing numbers of vacancies are randomly distributed in the plane that connects the two NCs. For a defect-free connection, the splitting energy reaches a value of around $35 \mathrm{meV}$. Increasing the number of vacancies, however, strongly reduces the splitting energy and diminishes the electronic coupling between the NCs by tens of meV. While vacancies are not identical to the defects leading to mosaicity in PbSe NC superlattices, the increase of electronic coupling upon reducing the vacancy density suggests that a reduced defect density is linked to an enhanced electronic coupling between NCs. The subsequent delocalization of the wave function is then observed through the optical and electronic properties of the NC solid. It is furthermore interesting to note that this type of disorder can be difficult to observe through TEM measurements, but is indirectly observed through X-ray measurements, as it contributes to the broadening of the diffraction peaks. Recent reports, together with the work presented here, attest to the growing awareness that engineering defect-free NC solids is crucial in obtaining highperformance NC devices and that the current less-than-expected performance can be improved through a broader understanding of the defects present in such structures. $(30,31)$ Here, we show that thermal annealing is a possible pathway to reduce defect densities without compromising the overall morphology of the NC superlattice. 
a
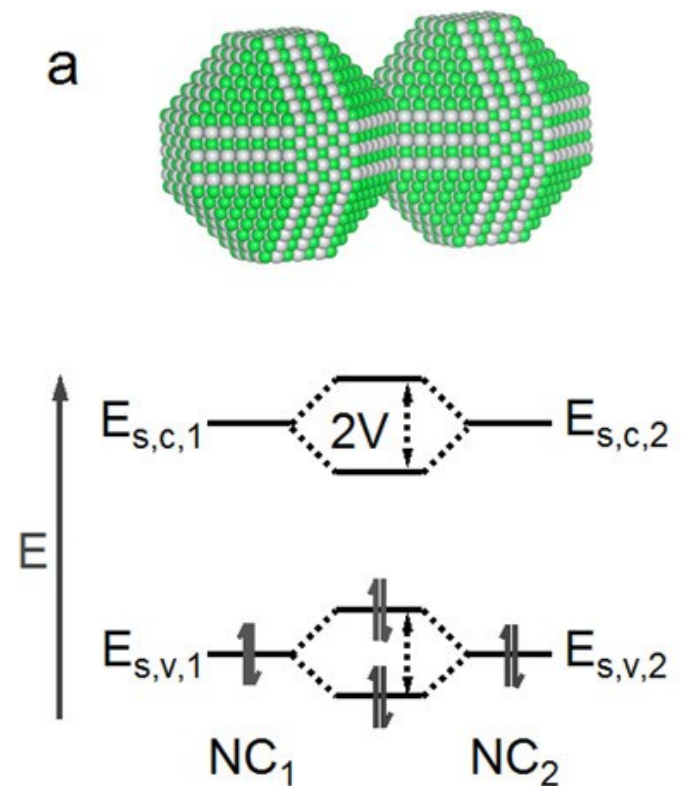

b

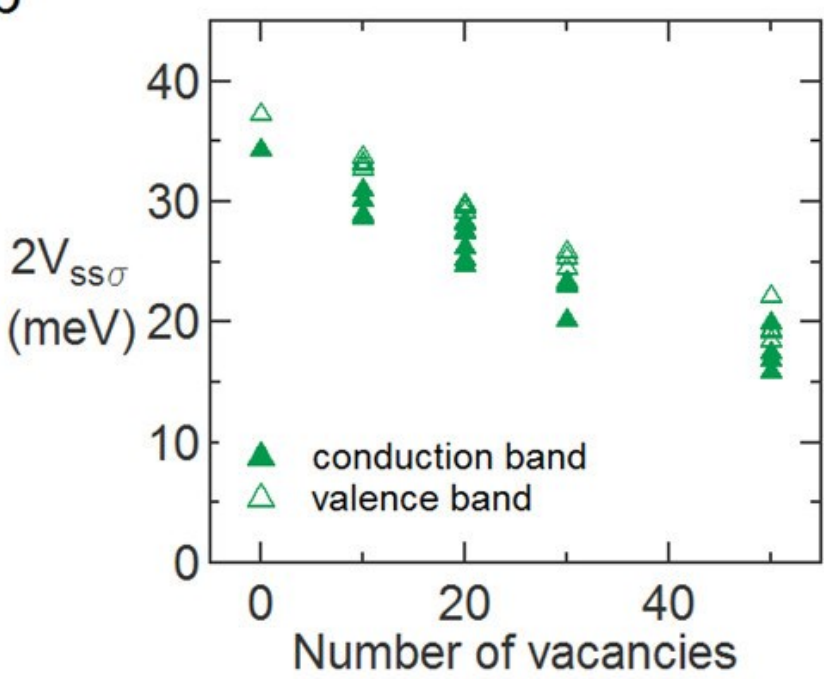

Figure 8. (a) A NC dimer model of two epitaxially connected NCs. Each NC is characterized by one $\mathrm{S}$ orbital in the valence band and one S orbital in the conduction band. Upon bonding, two dimer bonding and antibonding orbitals are formed, with the splitting energy between the two orbitals depending on the strength of the bond between the two NCs. (b) A plot showing the splitting energy $2 \mathrm{Vss} \sigma$ as a function of the amount of vacancies randomly distributed in the plane connecting both NCs. We find that the splitting energy-being a measure for the electronic coupling between the NCs-depends on the defect density in the epitaxial connection.

\section{Conclusion}

In this work, we experimentally link structural disorder in PbSe NC superlattices to delocalization of the wave function. The discrepancy between experimental observations and theoretical predictions of the NC solid properties, where, for example, expected band-edge red shifts of $>100 \mathrm{meV}$ compared to the colloidal NCs are in stark contrast with observed red shifts of $\sim 15-18 \mathrm{meV}$, formed the starting point to investigate thermal treatments of the room-temperature synthesized NC solids. We first established the thermal stability window through in situ GISAXS and in situ XRF measurements and find that the superlattices keep their structural integrity at temperatures ranging up to $\sim 300^{\circ} \mathrm{C}$. Annealing at temperatures well below this upper limit, for example, at $150{ }^{\circ} \mathrm{C}$, has no effect on the morphology of the superlattice and on the shape of the NCs, which we conclude from GISAXS and HR-TEM measurements. In this low-temperature regime, however, red shifts of the band-edge transition of $\sim 75 \mathrm{meV}$ compared to the colloidal NCs are seen, which brings the optical properties more in agreement with the predicted properties for NC solids with dispersive band and comparable relative neck widths. This is furthermore in line with observed changes in the electronic properties, where we find a $\sim 10$-fold enhancement of the charge mobility from $2.3 \times 10-3 \mathrm{~cm} 2 / \mathrm{V} \cdot \mathrm{s}$ to $1.9 \times 10-2 \mathrm{~cm} 2 / \mathrm{V} \cdot \mathrm{s}$ and a reduction of the hopping activation energy from 29 to $13 \mathrm{meV}$ after annealing for $1 \mathrm{~h}$ at $150^{\circ} \mathrm{C}$. Given the unchanged superlattice morphology and NC shape, we subsequently focused on structural disorder at the atomic scale. Here we find that even though there is long-range order on the level of the superlattice, still a substantial amount of disorder is present at the atomic level. From rocking curve XRD measurements and a Williamson-Hall size-strain analysis, we find that the atomic disorder mainly originates from NC mosaicity, leading to edge dislocations and point defects. Through gentle annealing of the NC superlattices, both the density of edge dislocations and point defects are reduced, pointing toward the connection between defect density and optoelectronic properties of the NC superlattices. We therefore conclude that the less-than- 
expected NC superlattice properties partially originate from faulty NC-NC interfaces; a result from the room temperature synthesis and the large amount of interfaces that are created during the formation of the NC superlattice. This work emphasizes the importance of understanding how structural disorder affects the optoelectronic properties of NC solids and provides a clear and consistent strategy to improve the performance of NC solids devices.

\section{Methods}

\section{Synthesis of Colloidal PbSe Nanocrystals}

Hydroxide-free lead oleate ( $\mathrm{Pb}$ (oleate)2) was synthesized from lead trifluoroacetate according to a previously reported method.(44) A solution of TOP-Se was prepared by dissolving $0.2021 \mathrm{~g}$ of Se powder $(2.56 \mathrm{mmol})$ and $25 \mu \mathrm{L}$ of diphenylphospine $(0.144 \mathrm{mmol})$ into $3.175 \mathrm{~mL}$ of trioctylphospine (7.12 mmol) at $150^{\circ} \mathrm{C}$. In a three-neck-flask, $0.616 \mathrm{~g}$ of $\mathrm{Pb}$ (oleate) 2 was dissolved into $4 \mathrm{~mL}$ of diphenylether and heated to $180^{\circ} \mathrm{C}$. At this temperature, $3 \mathrm{~mL}$ of the TOP-Se solution was injected, and the NCs were grown for $60 \mathrm{~s}$ at $150^{\circ} \mathrm{C}$. The temperature was set at $150^{\circ} \mathrm{C}$ right before injecting the TOP-Se. After $60 \mathrm{~s}$, the reaction mixture was quenched with $10 \mathrm{~mL}$ of butanol, and the synthesized NCs were precipitated by addition of $10 \mathrm{~mL}$ of acetonitrile to the reaction flask. After centrifugation, the supernatant was discarded, and the obtained NCs were washed three times by dispersion in toluene followed by precipitation with acetonitrile and centrifugation. The resulting 6.5 $\mathrm{nm}$ PbSe NCs were stored in toluene. The absorbance spectrum and a bright-field TEM image of the thus obtained NCs are shown in Supporting Information S1.

\section{Formation of Superlattices of Interconnected PbSe Nanocrystals}

Superlattices of PbSe NCs were made by first dropcasting $0.50 \mu \mathrm{L}$ of a $32 \mu \mathrm{M}$ solution of $6.5 \mathrm{~nm}$ NCs in toluene on the surface of $3.3 \mathrm{~mL}$ ethylene glycol contained in a glass vial with a $25 \mathrm{~mm}$ diameter. NC epitaxy was induced by injecting $1 \mathrm{~mL}$ of a $1 \mathrm{M}$ solution of aniline in ethylene glycol into the subphase. After reacting for $30 \mathrm{~min}$ at room temperature, the superlattices were transferred to a substrate of choice by Langmuir-Schaeffer deposition (stamping on glass microscope slides for optical absorption measurements, single polished Si for GISAXS, XRF, and XRD measurements, TEM grids for electron microscopy). After deposition, dry films were obtained by washing the superlattices twice by dipping in anhydrous acetonitrile for $20 \mathrm{~s}$.

\section{Tight-Binding Calculations}

For the theoretical modeling, we have considered 2D superlattices inspired from the experimental observations (Figures $1 \mathrm{~b}$ and $4 \mathrm{~b}$ ). The superlattices are made of a $2 \mathrm{D}$ periodic ensemble of tangential spherical NCs. The superlattice parameter is equal to the NC diameter $D(D=5.5 \mathrm{~nm})$. Each NC is connected to four others along the (001) directions. The neck between each pair of neighbor NCs is described by a cylinder of diameter $L$. The electronic structure of the superlattices is calculated in tight-binding at varying necking, that is, for different values of the ratio between $L$ and $D$. We assume that there is no relaxation of the PbSe lattice inside the NCs and the necks. Each Pb or Se atom is described by a double set of sp3d5s* atomic orbitals including the spin degree of freedom. The tightbinding parameters including spin-orbit coupling were taken from literature.(45) Due to the large number of atoms per unit cell, only the energy bands near the gap are calculated.

\section{In Situ GISAXS and XRF Measurements}

In situ GISAXS and XRF measurements during thermal annealing in He were carried out at the DUBBLE BM26B beamline(46) of the European synchrotron radiation facility (ESRF, Grenoble, France) in a dedicated setup.(47) The samples were positioned in a stainless steel chamber on a dedicated 
heating stage controlled via a Eurotherm PID regulator. After pumping down the chamber to $10-6$ mbar with the sample in place, He was injected into the chamber at a flow rate of $500 \mathrm{~cm} 3 / \mathrm{min}$ until a pressure of 1 bar was reached. Next, the annealing process was started while keeping the incoming gas flow constant at $500 \mathrm{~cm} 3 / \mathrm{min}$. Continuous GISAXS patterns were recorded with a Pilatus $1 \mathrm{M}$ detector from Dectris positioned at $4500 \mathrm{~mm}$ from the sample and using an acquisition time of $30 \mathrm{~s}$. The beam energy was $14 \mathrm{keV}$, and the incidence angle was set to $0.4^{\circ}$. The beam size at the sample position was set to $4 \mathrm{~mm} \times 1 \mathrm{~mm}(\mathrm{H} \times \mathrm{V})$. XRF spectra were simultaneously measured with a Vortex Si drift detector.

\section{Field-Effect Measurements}

Transistors were fabricated on highly doped p-type (100) Si substrates $(<0.005 \Omega \cdot \mathrm{cm})$ with $80 \mathrm{~nm}$ $\mathrm{SiO} 2$ grown by PECVD (Advanced Vacuum Vision-310). The SiO2 surface was passivated with $180 \mathrm{~nm}$ $B C B$ by spin coating a 1:2 BCB (Cyclotene 3022-35):mesithylene solution followed by post-annealing at $280^{\circ} \mathrm{C}$ in $\mathrm{N} 2$ atmosphere. Interdigitated Ti/Au contacts were fabricated by e-beam Ti/Au metal evaporation and subsequent lift-off. The metal fingers are $69 \mu \mathrm{m}$ long, $2 \mu \mathrm{m}$ wide, and $150 \mathrm{~nm}$ high with a $4 \mu \mathrm{m}$ pitch. Each device has two contact pads with a $200 \times 200 \mu \mathrm{m} 2$ area. To obtain a patterned NC superlattice deposition, microstructure patterns were defined by standard image reversal lithography and subsequent lift-off techniques. Ti-Prime adhesion promoter was first spincoated at $3000 \mathrm{rpm}$ and baked at $120^{\circ} \mathrm{C}$ for $3 \mathrm{~min}$. Then, AZ-5214 E resist was spin coated at 3000 $\mathrm{rpm}$ and baked at $100^{\circ} \mathrm{C}$ for $3 \mathrm{~min}$. After exposure through a contact mask using SUSS MA6 mask aligner, the patterns were developed with AZ-400 developer and cross-linked by post-baking at 120 ${ }^{\circ} \mathrm{C}$ for 2 min. After Langmuir-Schaeffer deposition (stamping) of the NC superlattice and removal of the photoresist by washing with anhydrous acetonitrile, the transfer curves were recorded in a nitrogen-filled glovebox using two Keithley 2450 source-measuring units.

The differential mobility is defined ....

\section{Temperature-Dependent Conductivity}

The temperature-dependent conductivity was measured through a four-probe measurement with 10 $\mu \mathrm{m}$ probe spacing. The sample was loaded in an in-house built cryostat setup and evacuated. The setup was cooled by circulating liquid nitrogen, resulting in a minimum temperature at the sample stage of $133 \mathrm{~K}\left(-140^{\circ} \mathrm{C}\right)$. After thermalization, the sample stage was gradually heated to room temperature through a Labview controlled temperature program. The average of 100 conductivity measurements was recorded every $5 \mathrm{~K}$ during the heating process.

\section{In Situ XRD Rocking Curve Measurements}

The samples were loaded into an annealing chamber mounted on a commercial Bruker D8 XRD system equipped with a CuKa X-ray source and goebel mirror. A linear Vantec detector was positioned with fixed angle relative to the $X$-ray source, enabling acquisition of the diffraction pattern in a single snapshot in a $2 \theta$ range of $23^{\circ}-36^{\circ}$ and with a resolution of $0.012^{\circ}$ per pixel. The out-ofplane orientation of the (002) diffraction plane of the NCs was investigated by changing the inclination of the incoming beam $\theta$ from $7^{\circ}$ to $22^{\circ}$ in $0.25^{\circ}$ steps while simultaneously changing the detector position in $0.5^{\circ}$ steps. The corresponding inclination angle of the probed diffracted plane was calculated for every step in $\theta$ and for every pixel. After loading the samples, the annealing chamber was immediately evacuated and filled with He-overpressure at a constant He-flow of $250 \mathrm{~cm} 3 / \mathrm{min}$. The orientation of the NCs was investigated both before and after isothermal anneal, without altering the samples position within the annealing chamber. The heating rates during this 
isothermal anneal were limited to $3^{\circ} \mathrm{C} / \mathrm{s}$, after which the sample remained at a temperature of $150{ }^{\circ} \mathrm{C}$ for $60 \mathrm{~min}$.

\section{Williamson-Hall Analysis}

We collected the X-ray data for the Williamson-Hall analysis at the A2 beamline at CHESS. We used photon energy of $11.22 \mathrm{keV}$ and recorded the diffraction signal with a PILATUS detector positioned $0.5 \mathrm{~m}$ away from the sample. The experiment was done in air. We collected reciprocal space data around three different reflections (002), (004), and (006). For each peak, we tuned the angle to the perfect Bragg condition and measured a single slice of the Ewald sphere. We subtracted the background from all diffraction patterns and fit the peak with two Gaussian functions. The Bragg peak width, especially for high order reflections, is significantly larger perpendicular to q direction than parallel to it. Taking this into account, we determined the width parallel to q from the 2D image as width on the Ewald sphere divided by $\cos \theta$. We studied one pristine and four superlattices annealed ex situ for $1 \mathrm{~h}$ at $75^{\circ} \mathrm{C}, 100^{\circ} \mathrm{C}, 125^{\circ} \mathrm{C}$, and $150^{\circ} \mathrm{C}$. For each film, we investigated four to five different locations on the sample. For each location we conducted the Williamson-Hall analysis. The data in the main text shows the result with the best linear fit for the widths as a function of $q$.

\section{Supporting Information}

The Supporting Information is available free of charge on the ACS Publications website at DOI: 10.1021/acsnano.9b04757.

The absorbance and TEM characterization of the NCs used in this work (S1); the morphology of PbSe NC superlattices deposited on cross-fingered gold electrodes (S2); HR-TEM observation of defective NC-NC interfaces (S3); the thermal stability of PbSe NC superlattices on Si substrates for different NC sizes and ligand coverages (S4.1); TEM images of annealed PbSe NC superlattices and analysis of connectivity (S4.2); TEM image of a large-area monocrystalline PbSe NC superlattice after annealing (S4.3); a TGA/DTA characterization of PbSe NCs (S5); the thermal stability of PbSe NC superlattices on ethylene glycol (S6); an analysis of the optical transitions in annealed NC superlattices (S7); and fieldeffect mobility measurements on NC superlattices (S8)

\section{Acknowledgments}

Z.H. acknowledges support by the European Comission via the Marie-Sklodowska Curie action Phonsi (H2020-MSCA-ITN-642656), the Marie-Sklodowska Curie action Compass (H2020-MSCA-ITN-2015691198), and IWT-Vlaanderen (SBO-MIRIS). C.D. and Z.H. acknowledge funding by BOF-UGent GOA nos. 01G01513 and 01G01019. C.D. acknowledges support by the European Comission via the MarieSklodowska Curie action HYCOAT. The Fund for Scientific Research Flanders (FWO-Vlaanderen) is recognized for financing beam time at the DUBBLE beamline of the ESRF and the associated travel costs. For research conducted at the Cornell High Energy Synchrotron Source (CHESS), J.P.C.R. acknowledges support from the National Science Foundation under award DMR-1332208. P.D. was supported through the Engineering Learning Initiatives Undergraduate Research Grants Program in the College of Engineering at Cornell University with funds from the Semiconductor Research Corporation (SRC) Education Alliance with support from the Intel Foundation. The authors thank Katrien Haustraete and Funda Alic for TGA/DTA measurements.

\section{References}


1Nie, Z.; Petukhova, A.; Kumacheva, E. Properties and Emerging Applications of Self-Assembled Structures Made from Inorganic Nanoparticles. Nat. Nanotechnol. 2010, 5, 15- 25, DOI: 10.1038/nnano.2009.453, , ,

2Sun, S.; Murray, C. B.; Weller, D.; Folks, L.; Moser, A. Monodisperse FePt Nanoparticles and Ferromagnetic FePt Nanocrystal Superlattices. Science 2000, 287, 1989-1992, DOI: 10.1126/science.287.5460.1989 , , ,

3 Hoinville, J.; Bewick, A.; Gleeson, D.; Jones, R.; Kasyutich, O.; Mayes, E.; Nartowski, A.; Warne, B.; Wiggins, J.; Wong, K. High Density Magnetic Recording on Protein-Derived Nanoparticles. J. Appl. Phys. 2003, 93, 7187-7189, DOI: 10.1063/1.1555896, ,

4Lee, J.; Hernandez, P.; Lee, J.; Govorov, A. O.; Kotov, N. A. Exciton-Plasmon Interactions in Molecular Spring Assemblies of Nanowires and Wavelength-Based Protein Detection. Nat. Mater. 2007, 6, 291-295, DOI: 10.1038/nmat1869 , , ,

5Lee, J.; Govorov, A. O.; Kotov, N. A. Nanoparticle Assemblies with Molecular Springs: A Nanoscale Thermometer. Angew. Chem., Int. Ed. 2005, 44, 7439- 7442, DOI: 10.1002/anie.200501264 , , 6Saran, R.; Curry, R. J. Lead Sulphide Nanocrystal Photodetector Technologies. Nat. Photonics 2016, 10, 81-92, DOI: 10.1038/nphoton.2015.280 , ,

7Ren, Z.; Sun, J.; Li, H.; Mao, P.; Wei, Y.; Zhong, X.; Hu, J.; Yang, S.; Wang, J. Bilayer PbS Quantum Dots for High-Performance Photodetectors. Adv. Mater. 2017, 29, 1702055, DOI:

10.1002/adma.201702055,

8Hu, C.; Gassenq, A.; Justo, Y.; Devloo-Casier, K.; Chen, H.; Detavernier, C.; Hens, Z.; Roelkens, G. Air-Stable Short-Wave Infrared PbS Colloidal Quantum Dot Photoconductors Passivated with Al2O3 Atomic Layer Deposition. Appl. Phys. Lett. 2014, 105, 171110, DOI: 10.1063/1.4900930 , , 9Yousefi Amin, A. A.; Killilea, N. A.; Sytnyk, M.; Maisch, P.; Tam, K. C.; Egelhaaf, H.-J.; Langner, S.; Stubhan, T.; Brabec, C. J.; Rejek, T.; Halik, M.; Poulsen, K.; Niehaus, J.; Köck, A.; Heiss, W. Fully Printed Infrared Photodetectors from PbS Nanocrystals with Perovskite Ligands. ACS Nano 2019, 13, 23892397, DOI: 10.1021/acsnano.8b09223 [ACS Full Text ACS Full Text],

10Yang, Z.; Fan, J. Z.; Proppe, A. H.; de Arquer, F. P. G.; Rossouw, D.; Voznyy, O.; Lan, X.; Liu, M.; Walters, G.; Quintero-Bermudez, R.; Sun, B.; Hoogland, S.; Botton, G. A.; Kelley, S. O.; Sargent, E. H. Mixed-Quantum-Dot Solar Cells. Nat. Commun. 2017, 8, 1325, DOI: 10.1038/s41467-017-01362-1 , , , 11Balazs, D. M.; Bijlsma, K. I.; Fang, H.-H.; Dirin, D. N.; Döbeli, M.; Kovalenko, M. V.; Loi, M. A. Stoichiometric Control of the Density of States in PbS Colloidal Quantum Dot Solids. Sci. Adv. 2017, 3, eaao1558, DOI: 10.1126/sciadv.aao1558, ,

12Zhang, J.; Xu, B.; Yang, L.; Ruan, C.; Wang, L.; Liu, P.; Zhang, W.; Vlachopoulos, N.; Kloo, L.; Boschloo, G.; Sun, L.; Hagfeldt, A.; Johansson, E. M. J. The Importance of Pendant Groups on Triphenylamine-Based Hole Transport Materials for Obtaining Perovskite Solar Cells with over 20\% Efficiency. Adv. Energy Mater. 2018, 8, 1701209, DOI: 10.1002/aenm.201701209 ,

13Hetsch, F.; Zhao, N.; Kershaw, S. V.; Rogach, A. L. Quantum Dot Field Effect Transistors. Mater. Today 2013, 16, 312- 325, DOI: 10.1016/j.mattod.2013.08.011 , ,

14Oh, S. J.; Berry, N. E.; Choi, J. H.; Gaulding, E. A.; Lin, H.; Paik, T.; Diroll, B. T.; Muramoto, S.; Murray, C. B.; Kagan, C. R. Designing High-Performance PbS and PbSe Nanocrystal Electronic Devices through Stepwise, Post-Synthesis, Colloidal Atomic Layer Deposition. Nano Lett. 2014, 14, 15591566, DOI: 10.1021/nl404818z [ACS Full Text ACS Full Text], ,

15Liu, Y.; Tolentino, J.; Gibbs, M.; Ihly, R.; Perkins, C. L.; Liu, Y.; Crawford, N.; Hemminger, J. C.; Law, M. PbSe Quantum Dot Field-Effect Transistors with Air-Stable Electron Mobilities above $7 \mathrm{~cm} 2 \mathrm{~V}-1 \mathrm{~s}-$ 1. Nano Lett. 2013, 13, 1578-1587, DOI: 10.1021/nl304753n [ACS Full Text ACS Full Text], , 16Gao, Y.; Aerts, M.; Sandeep, C. S. S.; Talgorn, E.; Savenije, T. J.; Kinge, S.; Siebbeles, L. D. a.; Houtepen, A. J. Photoconductivity of PbSe Quantum-Dot Solids: Dependence on Ligand Anchor 
Group and Length. ACS Nano 2012, 6, 9606-9614, DOI: 10.1021/nn3029716 [ACS Full Text ACS Full Text], ,

17Kim, G. H.; Garcia de Arquer, F. P.; Yoon, Y. J.; Lan, X.; Liu, M.; Voznyy, O.; Yang, Z.; Fan, F.; Ip, A. H.; Kanjanaboos, P.; Hoogland, S.; Kim, J. Y.; Sargent, E. H. High Efficiency Colloidal Quantum Dot Photovoltaics via Robust Self-Assembled Monolayers. Nano Lett. 2015, 15, 7691-7696, DOI: 10.1021/acs.nanolett.5b03677 [ACS Full Text ACS Full Text], ,

18André, A.; Theurer, C.; Lauth, J.; Maiti, S.; Hodas, M.; Samadi Khoshkhoo, M.; Kinge, S.; Meixner, A. J.; Schreiber, F.; Siebbeles, L. D. A.; Braun, K.; Scheele, M. Structure, Transport and Thotoconductance of PbS Quantum Dot Monolayers Functionalized with a Copper Phthalocyanine Derivative. Chem. Commun. 2017, 53, 1700-1703, DOI: 10.1039/C6CC07878H , , ,

19Anderson, N. C.; Hendricks, M. P.; Choi, J. J.; Owen, J. S. Ligand Exchange and the Stoichiometry of Metal Chalcogenide Nanocrystals: Spectroscopic Observation of Facile Metal-Carboxylate Displacement and Binding. J. Am. Chem. Soc. 2013, 135, 18536-18548, DOI: 10.1021/ja4086758 [ACS Full Text ACS Full Text], ,

20Evers, W. H.; Nijs, B. D.; Filion, L.; Castillo, S.; Dijkstra, M.; Vanmaekelbergh, D. Entropy-Driven Formation of Binary Semiconductor-Nanocrystal Superlattices. Nano Lett. 2010, 10, 4235- 4241, DOI: 10.1021/nl102705p [ACS Full Text ACS Full Text], ,

21Alimoradi Jazi, M.; Janssen, V. A. E. C.; Evers, W. H.; Tadjine, A.; Delerue, C.; Siebbeles, L. D. A.; van der Zant, H. S. J.; Houtepen, A. J.; Vanmaekelbergh, D. Transport Properties of a TwoDimensional PbSe Square Superstructure in an Electrolyte-Gated Transistor. Nano Lett. 2017, 17, 5238- 5243, DOI: 10.1021/acs.nanolett.7b01348 [ACS Full Text ACS Full Text], ,

22Walravens, W.; De Roo, J.; Drijvers, E.; ten Brinck, S.; Solano, E.; Dendooven, J.; Detavernier, C.; Infante, I.; Hens, Z. Chemically Triggered Formation of Two-Dimensional Epitaxial Quantum Dot Superlattices. ACS Nano 2016, 10, 6861-6870, DOI: 10.1021/acsnano.6b02562 [ACS Full Text ACS Full Text], ,

23Evers, W. H.; Schins, J. M.; Aerts, M.; Kulkarni, A.; Capiod, P.; Berthe, M.; Grandidier, B.; Delerue, C.; van der Zant, H. S. J.; van Overbeek, C.; Peters, J. L.; Vanmaekelbergh, D.; Siebbeles, L. D. A. High Charge Mobility in Two-Dimensional Percolative Networks of PbSe Quantum Dots Connected by Atomic Bonds. Nat. Commun. 2015, 6, 8195, DOI: 10.1038/ncomms9195 , , ,

24Whitham, K.; Yang, J.; Savitzky, B. H.; Kourkoutis, L. F.; Wise, F.; Hanrath, T. Charge Transport and Localization in Atomically Coherent Quantum Dot Solids. Nat. Mater. 2016, 15, 557-563, DOI: 10.1038/nmat4576, , ,

25Whitham, K.; Hanrath, T. Formation of Epitaxially Connected Quantum Dot Solids: Nucleation and Coherent Phase Transition. J. Phys. Chem. Lett. 2017, 8, 2623-2628, DOI:

10.1021/acs.jpclett.7b00846 [ACS Full Text ACS Full Text], ,

26Kalesaki, E.; Evers, W. H.; Allan, G.; Vanmaekelbergh, D.; Delerue, C. Electronic Structure of Atomically Coherent Square Semiconductor Superlattices with Dimensionality below Two. Phys. Rev.

B: Condens. Matter Mater. Phys. 2013, 88, 115431, DOI: 10.1103/PhysRevB.88.115431 , ,

27Kalesaki, E.; Delerue, C.; Morais Smith, C.; Beugeling, W.; Allan, G.; Vanmaekelbergh, D. Dirac

Cones, Topological Edge States, and Nontrivial Flat Bands in Two-Dimensional Semiconductors with a Honeycomb Nanogeometry. Phys. Rev. X 2014, 4, 011010, DOI: 10.1103/PhysRevX.4.011010 ,,

28Delerue, C. From Semiconductor Nanocrystals to Artificial Solids with Dimensionality below Two. Phys. Chem. Chem. Phys. 2014, 16, 25734-25740, DOI: 10.1039/C4CP01878H , , , 29Tadjine, A.; Delerue, C. Colloidal Nanocrystals as LEGO $^{\circledR}$ Bricks for Building Electronic Band Structure Models. Phys. Chem. Chem. Phys. 2018, 20, 8177-8184, DOI: 10.1039/C7CP08400E , , , 30Sanchez, A. M.; Gott, J. A.; Fonseka, H. A.; Zhang, Y.; Liu, H.; Beanland, R. Stable Defects in Semiconductor Nanowires. Nano Lett. 2018, 18, 3081- 3087, DOI: 10.1021/acs.nanolett.8b00620 [ACS Full Text ACS Full Text], , 
31Ondry, J. C.; Hauwiller, M. R.; Alivisatos, A. P. Dynamics and Removal Pathway of Edge Dislocations in Imperfectly Attached PbTe Nanocrystal Pairs; Towards Design Rules for Oriented Attachment. ACS Nano 2018, 12, 3178-3189, DOI: 10.1021/acsnano.8b00638 [ACS Full Text ACS Full Text], ,

32Steckel, J. S.; Yen, B. K. H.; Oertel, D. C.; Bawendi, M. G. On the Mechanism of Lead Chalcogenide Nanocrystal Formation. J. Am. Chem. Soc. 2006, 128, 13032-13033, DOI: 10.1021/ja062626g [ACS Full Text ACS Full Text], ,

33Chua, L. L.; Zaumseil, J.; Chang, J. F.; Ou, E. C. W.; Ho, P. K. H.; Sirringhaus, H.; Friend, R. H. General Observation of n-Type Field-Effect Behaviour in Organic Semiconductors. Nature 2005, 434, 194-199, DOI: 10.1038/nature03376 , ,

34Houtepen, A. J.; Hens, Z.; Owen, J. S.; Infante, I. On the Origin of Surface Traps in Colloidal II-VI Semiconductor Nanocrystals. Chem. Mater. 2017, 29, 752- 761, DOI:

10.1021/acs.chemmater.6b04648 [ACS Full Text ACS Full Text], ,

35Guyot-Sionnest, P. Electrical Transport in Colloidal Quantum Dot Films. J. Phys. Chem. Lett. 2012, 3, 1169-1175, DOI: 10.1021/jz300048y [ACS Full Text ACS Full Text], ,

36Gilmore, R. H.; Winslow, S. W.; Lee, E. M.; Ashner, M. N.; Yager, K. G.; Willard, A. P.; Tisdale, W. A. Inverse Temperature Dependence of Charge Carrier Hopping in Quantum Dot Solids. ACS Nano 2018, 12, 7741- 7749, DOI: 10.1021/acsnano.8b01643 [ACS Full Text ACS Full Text], ,

37Evers, W. H.; Goris, B.; Bals, S.; Casavola, M.; De Graaf, J.; van Roij, R.; Dijkstra, M.; Vanmaekelbergh, D. Low-Dimensional Semiconductor Superlattices Formed by Geometric Control over Nanocrystal Attachment. Nano Lett. 2013, 13, 2317-2323, DOI: 10.1021/nl303322k [ACS Full Text ACS Full Text], ,

38Yu, D.; Wang, C.; Guyot-Sionnest, P. n-Type Conducting CdSe Nanocrystal Solids. Science 2003, 300, 1277-1280, DOI: 10.1126/science.1084424 , , ,

39Deng, Z.; Jeong, K. S.; Guyot-Sionnest, P. Colloidal Quantum Dots Intraband Photodetectors. ACS Nano 2014, 8, 11707- 11714, DOI: 10.1021/nn505092a [ACS Full Text ACS Full Text], ,

40Moram, M. A.; Vickers, M. E. X-ray Diffraction of III-Nitrides. Rep. Prog. Phys. 2009, 72, 036502, DOI: 10.1088/0034-4885/72/3/036502, ,

41Leszczynski, M.; Teisseyre, H.; Suski, T.; Grzegory, I.; Bockowski, M.; Jun, J.; Porowski, S.; Pakula, K.; Baranowski, J. M.; Foxon, C. T.; Cheng, T. S. Lattice Parameters of Gallium Nitride. Appl. Phys. Lett. 1996, 69, 73-75, DOI: 10.1063/1.118123 , ,

42Krysko, M.; Sarzynski, M.; Domagała, J.; Grzegory, I.; Łucznik, B.; Kamler, G.; Porowski, S.; Leszczyński, M. The Influence of Lattice Parameter Variation on Microstructure of GaN Single Crystals. J. Alloys Compd. 2005, 401, 261- 264, DOI: 10.1016/j.jallcom.2004.10.077 , ,

43Metzger, T.; Höpler, R.; Born, E.; Ambacher, O.; Stutzmann, M.; Stömmer, R.; Schuster, M.; Göbel, H.; Christiansen, S.; Albrecht, M.; Strunk, H. P. Defect Structure of Epitaxial GaN Films Determined by Transmission Electron Microscopy and Triple-Axis X-Ray Diffractometry. Philos. Mag. A 1998, 77, 1013-1025, DOI: 10.1080/01418619808221225,

44Hendricks, M. P.; Campos, M. P.; Cleveland, G. T.; Jen-La Plante, I.; Owen, J. S. A Tunable Library of Substituted Thiourea Precursors to Metal Sulfide Nanocrystals. Science 2015, 348, 1226-1230, DOI: 10.1126/science.aaa2951 , , ,

45Allan, G.; Delerue, C. Confinement Effects in PbSe Quantum Wells and Nanocrystals. Phys. Rev.

B: Condens. Matter Mater. Phys. 2004, 70, 245321, DOI: 10.1103/PhysRevB.70.245321 , ,

46Portale, G.; Cavallo, D.; Alfonso, G. C.; Hermida-Merino, D.; van Drongelen, M.; Balzano, L.; Peters, G. W. M.; Goossens, J. G. P.; Bras, W. Polymer Crystallization Studies under ProcessingRelevant Conditions at the SAXS/WAXS DUBBLE Beamline at the ESRF. J. Appl. Crystallogr. 2013, 46, 1681- 1689, DOI: 10.1107/S0021889813027076, , 
47Dendooven, J.; Solano, E.; Minjauw, M. M.; Van de Kerckhove, K.; Coati, A.; Fonda, E.; Portale, G.; Garreau, Y.; Detavernier, C. Mobile Setup for Synchrotron Based In Situ Characterization During Thermal and Plasma-Enhanced Atomic Layer Deposition. Rev. Sci. Instrum. 2016, 87, 113905, DOI: 10.1063/1.4967711 , , , 